\title{
III-V compounds as single photon emitters
}

\author{
Wang Xu(王旭) $)^{1,2 \dagger}$, Xu Lei(徐蕾) ${ }^{1}$, Jiang Yun(姜芸 $)^{1}$, Yin Zhouyang(尹舟洋 $)^{1}$, \\ Chan Christopher C. S. ${ }^{3 \dagger}$, Deng Chaoyong(邓朝勇) ${ }^{1}$, and Taylor Robert A. ${ }^{4}$ \\ (1 Key Laboratory of Electronic Functional Composite Materials of Guizhou Province, \\ Guizhou University, Guiyang 550025, China) \\ (2 Guizhou Institute of Quantum Information and Big Data Applied Technology, Guiyang 550002, China) \\ (3 Department of Physics, The Hong Kong University of Science and Technology, Clear Water Bay, \\ Kowloon, Hong Kong 999077, China) \\ (4 Department of Physics, University of Oxford, Clarendon Laboratory, Parks Road, Oxford, OX1 3PU, UK)
}

\begin{abstract}
Single-photon emitters (SPEs) are one of the key components in quantum information applications. The ideal SPEs emit a single photon or a photon-pair on demand, with high purity and distinguishability. SPEs can also be integrated in photonic circuits for scalable quantum communication and quantum computer systems. Quantum dots made from III-V compounds such as InGaAs or GaN have been found to be particularly attractive SPE sources due to their well studied optical performance and state of the art industrial flexibility in fabrication and integration. Here, we review the optical and optoelectronic properties and growth methods of general SPEs. Subsequently, a brief summary of the latest advantages in III-V compound SPEs and the research progress achieved in the past few years will be discussed. We finally describe frontier challenges and conclude with the latest SPE fabrication science and technology that can open new possibilities for quantum information applications.
\end{abstract}

Key words: single photon emitters; solid-states; quantum dots; 2D materials

DOI: $\mathrm{xxxx}$ PACC: $\mathrm{xxxx}$

\section{Introduction}

A single photon emitter (SPE) is a device which either emits a single photon on demand or emits entangled photons at a relatively high repetition frequency [1]. The emission characteristics of an SPE are different to an attenuated laser or thermal light source for which the number of photons emitted in a given time will follow a Poisson distribution. SPEs follow sub-Poisson distribution and the second-order coherence function $g^{(2)}(0)$ sets the limit on the photon probability per pulse, in which a SPE has $0 \leqslant g^{(2)}(0)<1$. There are three major characteristic standards used to measure actual SPEs: i) The purity of single photons, which requires a small value of $g^{(2)}(0)$. ii) The maximum repetition frequency $f_{\max }$ together with the external quantum efficiency $\eta_{\text {total }}$, which can be enhanced by the coupling of SPEs to cavity modes and iii) the indistinguishability $F_{(0)}$ which demands the identical output photons [2]. Claiming "Quantum Supremacy" [3] is becoming rather important, therefore SPEs are of great interest as they form central building blocks in a range of proposed quantum information schemes, including quantum computational realizations [4-6], precision measurement [7] and quantum secure communication schemes [8,9].

It is generally believed that a cold atom is naturally a single photon source [10,11]. However control atomic emission is rather difficult, and hard to scale [12]. Some new attempts at realizing interactions of photons, magnons and matter by way of single atoms are underway. For instance, a high precision electrometer was recently fabricated, using the Schrödinger's cat state with a single atom in a superposition of two circular Rydberg states [13]. The other major choices of SPEs include trapped ions [14-16], single quantum dots (QD) [17], nitrogen vacancy centers [18] in diamond, organic molecules [19], carrier localization centers in 2D materials [20-22], and recently carbon nanotubes $[23,24]$.

$\dagger$ Corresponding author. Email: xuwang@gzu.edu.cn, ccschan@ust.hk

Received March 2019

(c) 2019 Chinese Institute of Electronics 
Many studies have focused on SPEs, but as yet a perfect triggerable high purity, bright and indistinguishable SPE has not been proposed, especially one operating at room temperature. Amongst all the potential central blocks to build an optimal SPE, semiconductor single QD SPE systems have been studied for nearly two decades and have recently been proved to outperform the long-applied SPEs based on spontaneous parametric down-conversion (SPDC) [25, 26]. A quantum dot is an "atom like"three-dimensional confinement nano-sized area in a semiconductor. This is very different from the $2 \mathrm{D}$ graphene and $1 \mathrm{D}$ quantum wires due to its 3-dimensional confinement leading to a discrete density of states [27]. Sub-Poissonian light emission has been observed via fluorescence experiments [28, 29]. Quantum confinement of single particles in the quantized levels and Coulomb interaction determine the confined energy [30]. QD SPEs mainly include III-V InGaAs [31], GaAs [32] and InP [33] QDs systems. They offer significant scalability and can be integrated with state-of-the-art semiconductor technology [34]. II-VI Telluride or selenide QDs offer bright single-photon emission under room-temperature, but suffer from blinking phenomena [35, 36]. Here we review on the progress of SPEs based on III-V compound systems. Besides the QDs we just discussed, the scope of this review will also expand to hexagonal boron nitride (hBN) single layer, as an emerging potential 2D III-V SPEs [37].

\section{Several fundamental features of SPEs}

\subsection{Purity and Indistinguishability}

An ideal single photon emitter should, at a given time, emit one photon or an entangled photon pair with a certain repetition rate, which can be characterized by the aforementioned second-order intensity correlation function $g^{(2)}(0)$ measured by the Hanbury Brown and Twiss (HBT) experiment [38] as shown in Fig 1b. After sending a pulse of light to a beamsplitter, two detectors at each output sum up the coincidence correlations as a function of photon delay. This gives:

$$
g^{(2)}(0)=\frac{\left\langle n_{1}(t) n_{2}(t)\right\rangle_{t}}{\left\langle n_{1}(t)\right\rangle_{t}\left\langle n_{2}(t)\right\rangle_{t}}
$$

where $n_{\mathrm{i}}(t)$ is the number of counts from a given single photon detector at time $t$. Therefore, an optimal SPE has $g^{(2)}(0)=0$ as no coincidences should be seen when the delay is zero as a single photon would appear in one detector only. Fig 1d shows an example of a pulsed HBT trace taken from reference [39]. To keep the phase stability of the light with high single photon purity, the coherence time $T_{2}$ is of significant importance, with:

$$
\gamma=\frac{1}{T_{2}}=\frac{1}{2 T_{1}}+\frac{1}{T_{2}^{*}}
$$

where $\gamma$ is the photon line width, $T_{1}$ indicates the transition radiative lifetime and $T_{2}^{*}$ corresponds to the pure dephasing time. The QD transition should be short-lived with a high exciton recombination rate [40,41]. Therefore the environmental fluctuations greatly affects the quality of a SPE, especially when we are discussing III-V semiconductors as they generally possess large optical dipoles, which make them easier to couple with optical modes and their environment [17]. Another necessary characteristic of a SPE is indistinguishability. The Hong-Ou-Mandel (HOM) experiment tests the distinguishability of a system by sending two pulses into a beamsplitter and detects at each output of the coincidences seen, which can be characterized by the HOM visibility $V_{\mathrm{HOM}}$ as shown in Fig 1c [42]. If photons in the two pulses have the same spectral bandwidth, pulse width, polarization, carrier frequency and mode profile, then we call them indistinguishable photons with $V_{\mathrm{HOM}}=1$. In a more general case, $V_{\mathrm{HOM}}$ can be expressed by $\mathrm{M}$, as $M=V_{\mathrm{HOM}}\left(\left(R^{2}+T^{2}\right) / 2 R T\right)$ where $R=1-T$ and $R$ and $T$ are the intensity reflectivity and transmission coefficients of the beamsplitter, respectively [43]. Decoherence, photon purity and indistinguishability are all correlated and have direct application in quantum communications [44] and quantum error correction [45].

\subsection{Brightness and Scalability}

There are several descriptions to explain brightness in SPEs various from literature and different applications [46]. However, in general the brightness exhibits the portion of vacuum in a stream of light. The higher the brightness 


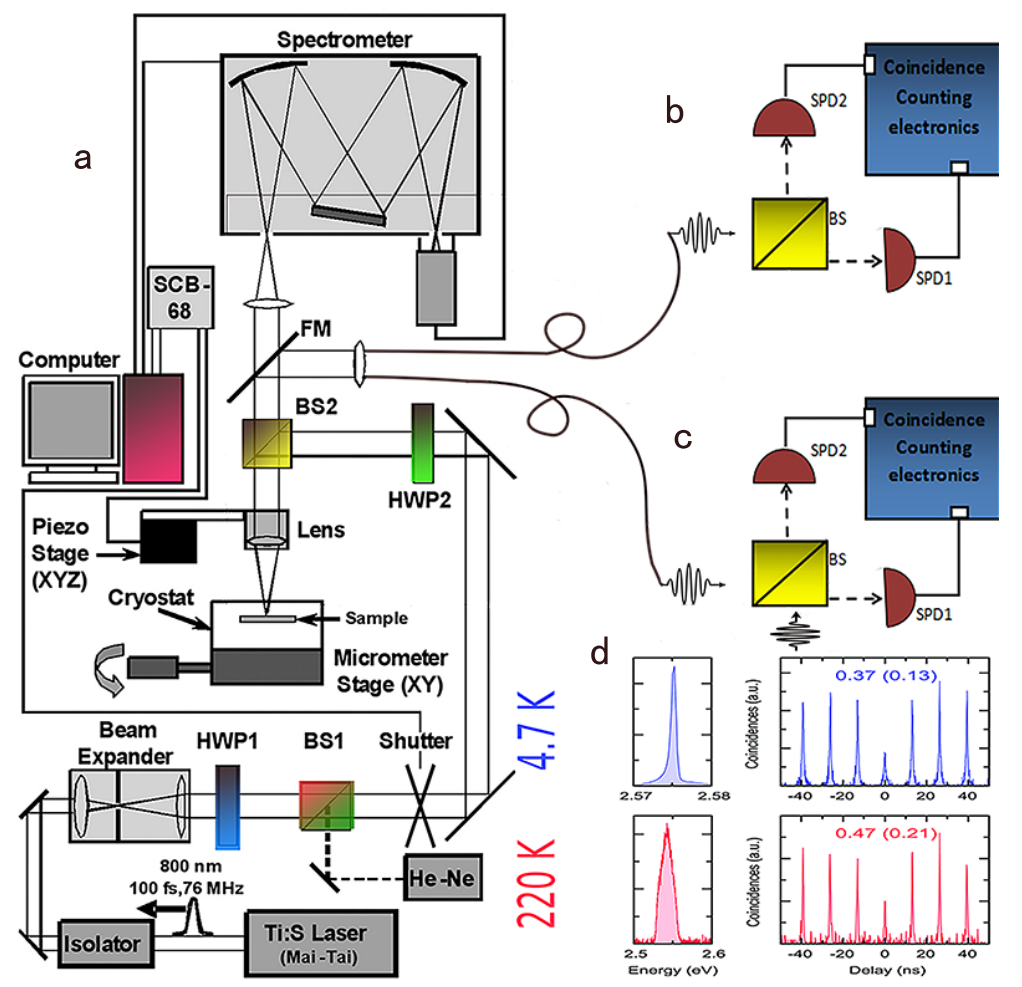

图 1: (a) Schematic diagram of the system used to perform general QDs micro-photoluminescence spectroscopy. (b) HBT experiment set-up. (c) HOM experiment set-up. (d) Examples of HBT experiment from [39].

of a SPE, the more consecutive of the single photons in a pulse. Here in this particular discussion we express the brightness as $B_{\text {lens }}$, measuring single photons collected at the first lens as a function of the counts in excitation pulse. Fig 1a shows a general QD micro-photoluminescence spectroscopy set-up. Brightness is directly related to singlephoton generation efficiencies and thus correlated to scalability of a system. This efficiency can be greatly enhanced by coupling to various optical cavities by employing cavity quantum electrodynamics. Such cavities include photonic crystal waveguides [47], photonic trumpets [48], nanowires [49], microlenses [50], bull's eye cavities [51], distributed Bragg gratings [32], and micropillars, which have achieved the highest extraction efficiency so far reported [52, 53].

\subsection{Single entangled photon-pair generation}

The famous EPR paper left us with many legacies, one of which stands at an intriguing position in quantum physics-the entanglement [54]. The quantum state of two entangled particles cannot be factorized in terms of the product of each single-particle wave function, which implies "spooky action at a distance", as coined by Einstein. Entanglement has tremendous applications in various quantum information processing protocols including quantum repeaters [55], quantum teleportation [56] and quantum simulations [57]. In a similar manner to SPEs, brightness, on-demand generation and indistinguishability are required for an entangled photon source. Entanglement fidelity is also on the checklist as the photon signal should be in a maximally entangled Bell state [58].

All the above conditions form a blueprint for an optimal SPE. There are also other desirable needs to be considered such as room temperature operation. In the following sections we examine SPEs in III-V compounds in detail and discuss their single photon emission properties from the point of view of material and physical considerations. 


\section{Single photon emission with III-V compounds}

\subsection{III-Asenides}

\subsubsection{QD growth}

In III-V compounds such as GaAs, InP and InAs, the different lattice parameters of the constituent components result in a lattice mismatch and strain at the interface [59]. This build-up strain becomes energetically favourable for the adatoms to form 3D-like structures beyond a certain critical thickness. InAs/GaAs QDs are obtained by straindriven nucleation in the Stranski-Krastanov (S-K) growth mode [60]. In these low energy interface structures, the strain energy increases with the thickness of the epilayers. Given the self-assembled nature of the S-K mode, the fabricated QDs are located randomly. A GaAs cap on top of the dots is used to enable bright emission and can also produce a redshift in the QD emission energy. Note a sacrificial AlGaAs layer fabricated above the GaAs buffer and below the dots is necessary for the production of an air bridge in the following cavity fabrication processes [61]. Various growth schemes have been adopted with different epilayer thickness or cap-layers such as InGaAs caps, in order to optimize the optical performance of the QDs [47]. Other growth methods are also applicable, for instance bottom-up-grown site-selected InAsP in nanowire was demonstrated with bright tunable SPEs [62]. Schweickert et al. obtained a high purity QD SPE by Al-droplet etching on $\mathrm{Al}_{0.4} \mathrm{Ga}_{0.6}$ As followed by deposition of $2 \mathrm{~nm}$ GaAs [63]. This method is of great importance as it produces highly-symmetric QDs, which play a key role in reducing the fine structure splitting (FSS) when fabricating entangled photon pair sources [64].

\subsubsection{Material properties}

The atom-like discrete nature of the energy levels generates promising interband transitions and is ascribed to the origin of single photon emission from QDs. As in the simplest picture of the energy levels shown in Fig. 2, electrons with spin $-1 / 2$ and heavy holes with spin $-3 / 2$ populate the conduction band (with s-like orbital symmetry) and valence band (with p-like symmetry), respectively. Light holes are neglected in this picture because they are pushed down in energy due to the confinement energy scales inversely with mass. Transitions are allowed when the overall symmetry of the two states are different, such as the transition $(\mathrm{p}, \mathrm{S}) \leftarrow \rightarrow(\mathrm{s}, \mathrm{S})$, denoted with 1X. Excitons are formed due to Coulomb interactions between the carriers. The so-called dark excitons in contrast with bright ones cannot recombine optically due to quantum selection rules. The transition $(\mathrm{p}, \mathrm{S}) \leftarrow \rightarrow(\mathrm{s}, \mathrm{P})$ is not allowed [65]. However, in processes such as two photon absorption (TPA), the dark transition can be observed. As more carriers are excited into the QD, a second exciton can be created, which can interact with the first forming a state known as a biexciton (XX) as illustrated in Fig. 2b. The linear dependence of the exciton emission intensity on excitation power becomes quadratic for a XX since every transition involves two electon-hole pairs. The repulsive Coulomb interaction between excitons and biexcitons leads to a decrease in the binding energy with increasing exciton transition energy, enabling the generation of entangled photon pairs.

Non-resonant excitation creates many electron-hole pairs in the material matrix surrounding the QDs, which then relax to the lowest energy confined states in the QDs [66]. This process is easier to realize as the pump energy is spectrally selected to differ from that of the emitted photons. However, multi-exciton generation greatly damages the coherence of the emitted photons and introduces time jitter, which has a detrimental effect on the indistinguishability [67]. Therefore, resonant pumping has now been widely applied instead. This process directly excites the QD into the s-shell and significantly diminishes the dephasing and time jitter, but is much more challenging experimentally.

\subsubsection{Single photons from GaAs based dots}

Emission from single III-As QDs was initially demonstrated using photoluminiscence in 1994 [68, 69]. The first quantized light was detected by Michler et al, using a Purcell enhanced high Q cavity generated excitonic transition with a single photon repetition rate of $82 \mathrm{MHz}$ [70] as shown in Fig. 3b. The brightness can be easily improved up to $10-20 \%$ by coupling the QD layer into a distributed Bragg reflector (DBRs) to form a 1D cavity (generally called planar cavity) [71]. The performance of other cavity enhanced devices will be discussed later. Several schemes 


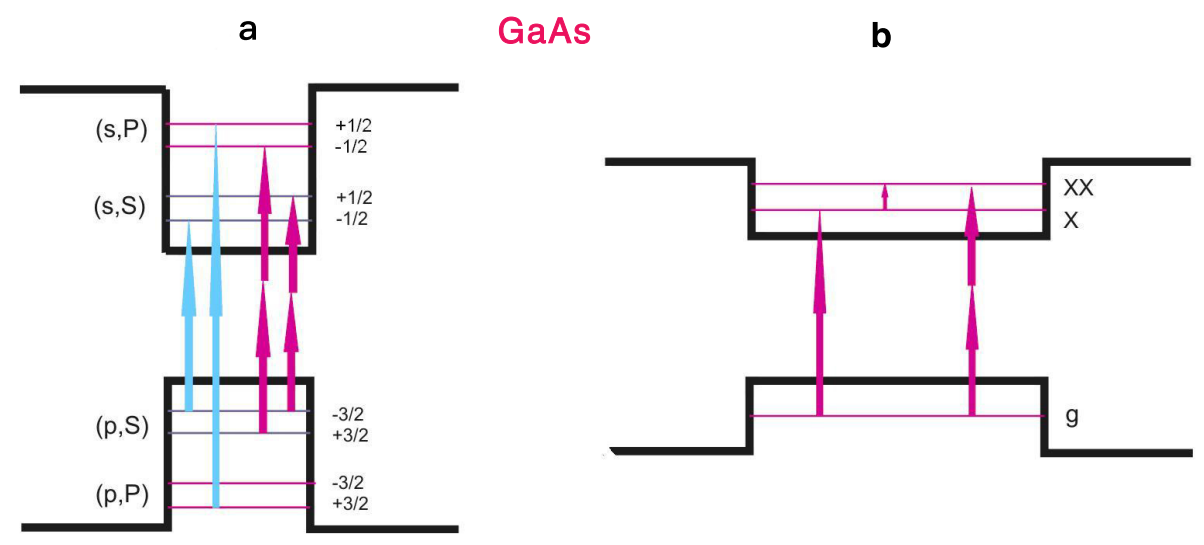

图 2: Simplified schemes of optical transitions from different single photon sources. (a) Electron and hole confined states in a QD. The left indices show the band and envelope orbital symmetries, respectively. The right indices indicate the spin states. (b) Electron and hole confined states in a bigger QD compared with (a). Excitons and biexcitons are indicated. It should be noted that only absorption is illustrated in (a) and (b).

with high extraction efficiency of over $70 \%$ were reported $[48,49,52,53,72]$. Due to the aforementioned relationship between brightness and scalability, purity and indistinguishability, increasing incident power leads to multi-cascade emissions and diminishes the coherence, under non-resonant excitation [73]. Therefore recent research has concentrated on using resonant s-shell excitation $[32,47,53,63]$ as shown in Fig. 3c and e. Somaschi et al. fabricated a connected micropillar cavity embedding an electro-driven InGaAs QD layer, emitting single photons with an indistinguishability of $0.9956 \pm 0.0045$ and a $g^{(2)}(0)=0.0028 \pm 0.0012$ [26]. Ding et al. deterministically generated resonance fluorescence single photons with an extraction efficiency of $0.66, g^{(2)}(0)=0.009 \pm 0.002$, and photon indistinguishability of $0.978 \pm 0.004$ [25]. However, $B_{\text {lens }}$ under resonant excitation is generally low as the wave packet of incident laser overlaps with single photon signal. Recently, a new method to extract the laser background without sacrificing the system efficiency was developed, and produced a SPE simultaneously exhibiting high brightness ( 0.7), high purity $(\sim 0.05)$ and an indistinguishability of $0.976 \pm 0.001$ [74].

\subsubsection{Coupling with cavities}

It is worth mentioning that the SPE brightness for III-As QDs can generally be modified and controlled by placing mirrors or conductors around the source, hence causing the density of states of the vacuum fluctuations to change. Above we just discussed the coupling with DBRs [77]. The performance can be further increased by the use of an optical antenna [48] or even just a backside gold mirror [78]. There are two distinct coupling regimes, in weak coupling the Purcell effect is seen. Here the coupling strength of light-matter interaction $g \propto Q / V$ defines the SPE performance and initially increasing the spontaneous emission was the main pursuit. Spontaneous emission was found to be weakly enhanced by Tanaka et al. using one-dimensional semiconductor microcavities [79]. A much stronger emission rate was found using three-dimensional confinement techniques, like that measured by Bayer [80]. An alternative method uses photonic band structures [81]. In this weak coupling regime, the coupling of a single emitter to the cavity can either enhance or inhibit the irreversible spontaneous emission rate due to the cavity Purcell effect [82]. Strong coupling on the other hand depends on $Q / \sqrt{V}$ and is very interesting as it allows non-linear quantum optics experiments to be performed in conditions of as few as two photons [83] and enables the optimization and control of source and dissipation [84]. Both single photon purity and indistinguishability were enhanced by coupling to various cavities as shown in Fig. 4. Amongst which, Liu et al. realized a radiative lifetime of $22.7 \mathrm{ps}$ in a waveguide-coupled quantum dot-photonic crystal cavity system [47] as shown in Fig. 4d. By combining a two-photon excitation process and a filtering and detection setup, Schweickert et al. produced a $g^{(2)}(0)$ of $0.000075 \pm 0.000016$, yielding the lowest value of a raw second-order correlation yet reported, to the best of our knowledge [63]. 

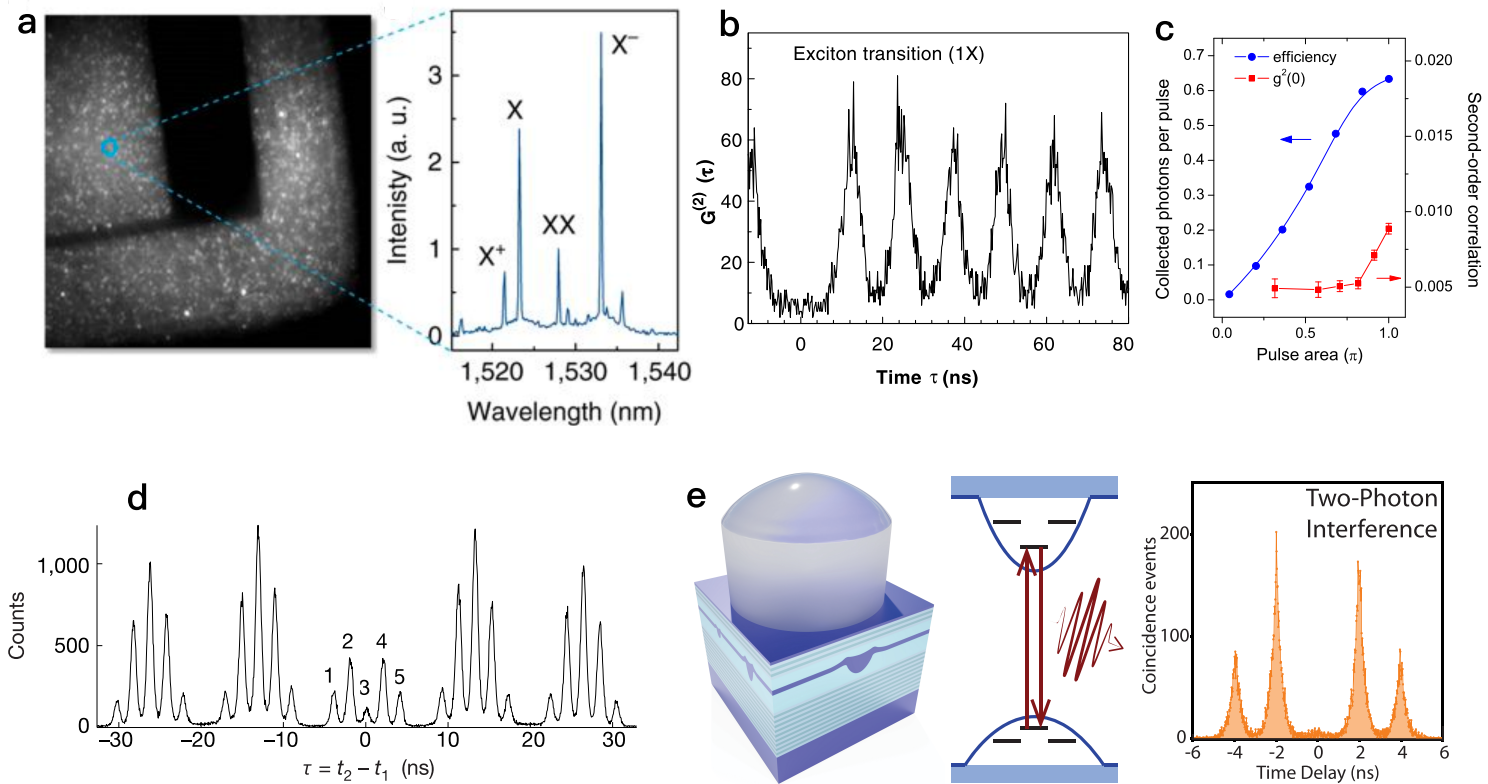

图 3: (a) Image of the bright spots showing individual QDs taken with an InGaAs camera and spectrum of the QD circled in a with exciton $(\mathrm{X})$, biexciton $(\mathrm{XX})$, positively charged exciton $\left(\mathrm{X}^{+}\right)$and negatively charged exciton $\left(\mathrm{X}^{-}\right)$ labelled [75]. (b) The measured unnormalized correlation function $g^{(2)}(0)$ [70]. (c) The comparison of photon extraction efficiency with pump power and photon purity from [25]. (d) Two-photon interference demonstrated from the small area of peak 3 [76]. (e) Resonance fluorescence of GaAs Quantum dots with near-unity photon indistinguishability [32].

\subsubsection{Generation of entangled photons}

In a similar manner to the general SPE that we just discussed above, generating entangled photons also requires that several conditions are met, including on-demand generation, high indistinguishability and high extraction efficiency (here efficiency extends to the product of extraction efficiency and pair generation efficiency). The source should produce maximally entangled Bell states, characterised by the fidelity. Very recently, following the fabrication of deterministic high symmetry QDs devices [87, 88], a SPE which simultaneously possesses all the four conditions has been produced. Chen et al. built a broadband optical antenna with a photon pair efficiency of $0.372 \pm 0.002$ and a fidelity of 0.9 [89]. Wang et al. generated entangled photon pairs with a fidelity of 0.9 , pair generation rate of $0.59 \pm 0.01, g^{(2)}(0)$ of $0.014 \pm 0.001$ and simultaneously with photon indistinguishability of $0.90 \pm 0.01$ [85]. Liu et al. even improved this photon count rate to $>10 \mathrm{MHz}$ [90], realizing an unprecedented brightness of photon-pair generation as high as 0.65 (single-photon efficiency of 0.85 ).

All the above discussions are summarized in Fig 6 generated from Table 1. State of the art technique allows for the production of SPEs with simultaneously high brightness, purity and indistinguishability. There are two exceptions worth mentioning, as i) the general trend is that both the single photon purity and indistinguishability decrease with increasing brightness as indicated by the red-dotted simulated line. The work of Schweickert et al. is an exception in that they greatly reduced the dark current [63] and resulted in high single photon purity. ii) Resonant excitation generally produced low brightness sources as it is difficult to remove laser background, however recent technique have improved this performance as shown in the line connected experiments in Fig 6 [74,90].

\subsubsection{Deterministic fabrication of SPEs}

When considering scalability of SPE integrated systems, the light emitter has to be placed at the antinode of the cavity, which also increases the device efficiency. The main issue then becomes how to couple the generated photons into well-defined spatial modes and out of the solid state cavity quantum electrodynamic devices [91]. To fabricate 

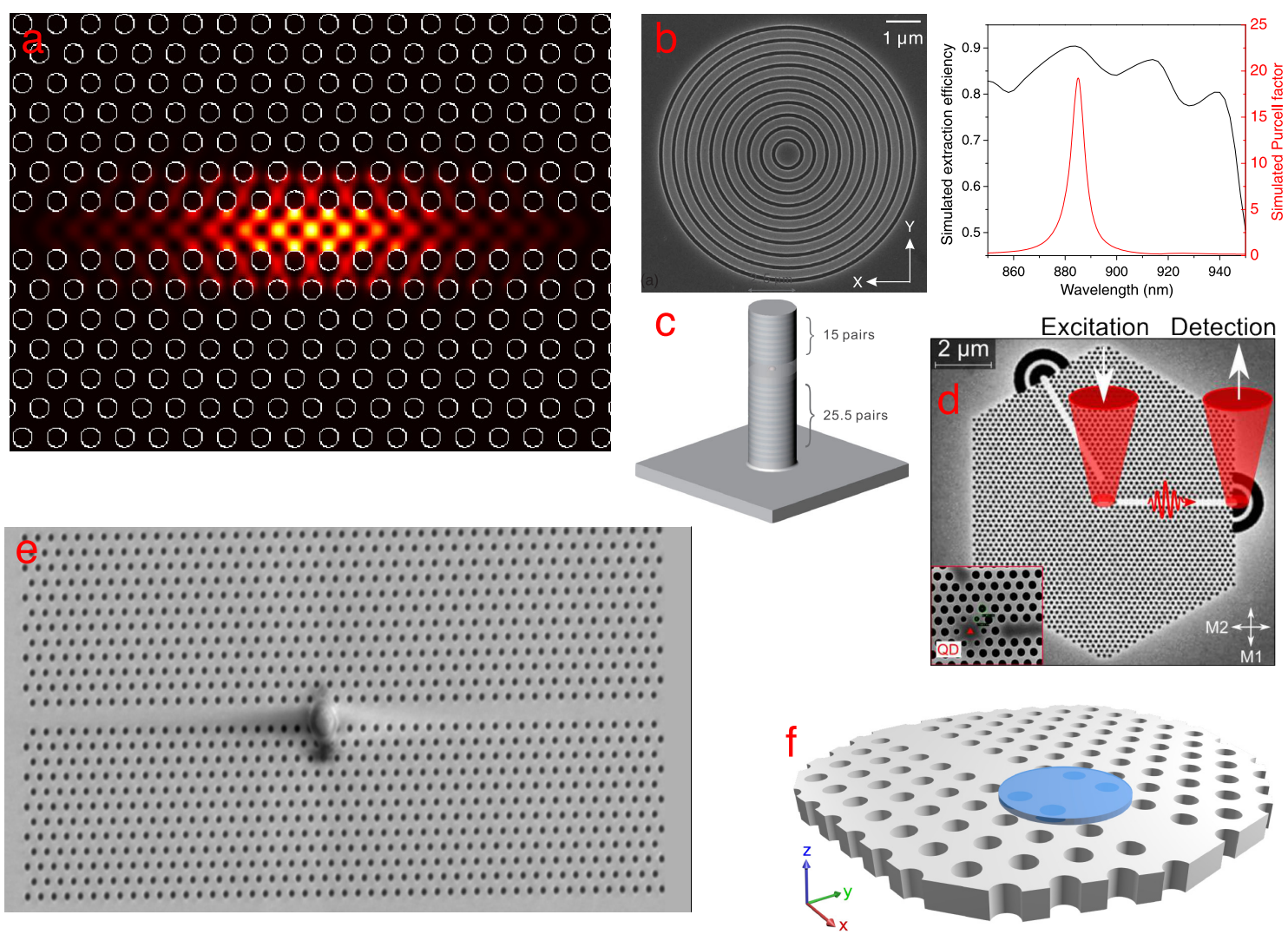

图 4: (a) Simulation of the electromagnetic field of a crystal photonic waveguide. (b) Microstructure of a bull's eye cavity and simulation of the single-photon extraction efficiency and Purcell factor as a function of photon emission wavelength of the cavity [85]. (c) Microplillar cavity used in [25]. (d) Schematic diagram of the waveguide-coupled quantum dot-photonic crystal cavity system [47]. (e) and (f) illustrated a mode-gap cavity depicted in [86]. 


\begin{tabular}{|c|c|c|c|c|c|c|c|c|c|c|}
\hline Reference & Source & $\begin{array}{l}\text { Photonic } \\
\text { structure }\end{array}$ & $\begin{array}{l}\text { Wavelength } \\
(\mathrm{nm})\end{array}$ & Lifetime (ns) & $\begin{array}{l}\text { Operation } \\
\text { temperature }\end{array}$ & Excitation & $\mathrm{B}_{\text {lens }}$ & $\mathrm{g}^{(2)}(0)$ & M & $\begin{array}{l}\text { Entanglement } \\
\text { fidelity }\end{array}$ \\
\hline [52] (2013) & InGaAs & Micropillar & 931 & $0.265-0.270$ & $10 \mathrm{~K}$ & Non-resonant & $\begin{array}{l}0.79 \pm 0.08 \\
0.53 \pm 0.05\end{array}$ & 0.05 & $\begin{array}{l}0.55 \pm 0.05 \\
0.92 \pm 0.10\end{array}$ & \\
\hline [72] (2015) & InGaAs & $\begin{array}{l}\text { Adiabatic } \\
\text { pillar }\end{array}$ & 945 & $0.14 \pm 0.04$ & $20 \mathrm{~K}$ & Non-resonant & $0.74 \pm 0.05$ & $\begin{array}{c}0.10 \\
\pm 0.03\end{array}$ & $0.75 \pm 0.05$ & \\
\hline$[50](2015)$ & InGaAs & Microlens & 932 & $\sim 1$ & $6 \mathrm{~K}$ & Non-resonant & $0.23 \pm 0.03$ & $<0.01$ & $0.80 \pm 0.07$ & \\
\hline [51] (2015) & InGaAs & $\begin{array}{l}\text { Bulls-eye } \\
\text { cavities }\end{array}$ & 907 & 0.52 & $6 \mathrm{~K}$ & Non-resonant & $0.48 \pm 0.05$ & $0.009 \pm 0.005$ & & \\
\hline [73] (2016) & InGaAs & Micropillar & 892.6 & 0.162 & $4.3 \mathrm{~K}$ & Non-resonant & 0.334 & 0.027 & 0.921 & \\
\hline [26] (2016) & InGaAs & $\begin{array}{l}\text { Connected } \\
\text { pillar }\end{array}$ & $\begin{array}{l}890 \mathrm{w} / \text { electrical } \\
\text { tuned }\end{array}$ & $0.08-0.12$ & $4 \mathrm{~K}$ & Resonant & $0.154 \pm 0.015$ & $0.0028 \pm 0.0012$ & $\begin{array}{c}0.989 \pm 0.004 \\
0.9956 \pm 0.0045\end{array}$ & \\
\hline$[25](2016)$ & InGaAs & Micropillar & 897.44 & 0.084 & $10 \mathrm{~K}$ & Resonant & 0.33 & $0.009 \pm 0.002$ & $\begin{array}{c}0.959 \pm 003 \\
0.978 \pm 0.004\end{array}$ & \\
\hline [114] (2017) & $\mathrm{GaN}$ & $\begin{array}{l}\text { Gallium nitride } \\
\text { crystal }\end{array}$ & $1085-1340$ & $0.736 \pm 0.004$ & Room & Non-resonant & & $0.05 \pm 0.02$ & & \\
\hline [123] (2017) & InGaN & N/A & 420.5 & 0.156 & $130 \mathrm{~K}$ & Non-resonant & & 0.18 & & \\
\hline [47] (2018) & InGaAs & $\begin{array}{c}\text { Photonic } \\
\text { crystal cavities }\end{array}$ & 915 & $0.0227 \pm 0.0009$ & $4 \mathrm{~K}$ & Resonant & 0.41 & $0.026 \pm 0.007$ & $0.939 \pm 0.033$ & \\
\hline [126] (2018) & $\mathrm{hBN}$ & $\begin{array}{c}\text { Plasmonic nanocavity } \\
\text { arrays }\end{array}$ & 566.04 & 0.375 & Room & Non-resonant & $0.5347^{*}$ & $0.033 \pm 0.047$ & & \\
\hline [63] (2018) & GaAs & Low-Q planar cavity & 793 & 0.125 & $4 \mathrm{~K}$ & Resonant & 0.5 & $0.000075 \pm 0.000016$ & & \\
\hline [135] (2018) & InAsP & Tapered InP nanowire & 1255 & 1.3 & $4 \mathrm{~K}$ & Non-resonant & 0.28 & 0.03 & & \\
\hline [128] (2018) & $\mathrm{hBN}$ & N/A & 660 & & Room & Non-resonant & & 0.3 & & \\
\hline [74] (2019) & InGaAs & Micropillar & 874 & & $1.5 \mathrm{~K}$ & Resonant & $\sim 0.7$ & $0.05 \pm 0.02$ & $0.976 \pm 0.001$ & \\
\hline [32] (2019) & GaAs & DBR & 789 & $0.196 \pm 0.002$ & $5 \mathrm{~K}$ & Resonant & $0.2 \pm 0.032$ & $(0.0025 \pm 0.0002)$ & 0.95 & \\
\hline [88] (2018) & GaAs & $\begin{array}{c}\text { Broadband } \\
\text { optical antenna }\end{array}$ & $780.3,781.6$ & $<0.2$ & $4 \mathrm{~K}$ & Resonant & 0.372 & $0.002 \pm 0.002$ & & 0.9 \\
\hline [90] (2019) & GaAs & $\begin{array}{l}\text { Bragg grating bull's- } \\
\text { eye cavity }\end{array}$ & 770,772 & 0.06 & $3.2 \mathrm{~K}$ & Resonant & $0.65 \pm 0.04$ & $0.001 \pm 0.001$ & $0.901 \pm 0.003$ & $0.88 \pm 0.02$ \\
\hline [89] (2019) & InGaAs & $\begin{array}{l}\text { Bragg grating bull's- } \\
\text { eye cavity }\end{array}$ & $879.4,881$ & 0.0664 & $4 \mathrm{~K}$ & Resonant & $0.59 \pm 0.01$ & $0.014 \pm 0.001$ & $0.9 \pm 0.01$ & $0.9 \pm 0.01$ \\
\hline
\end{tabular}

图 5: * denotes the brightness of hBN after transfer comparing to its original brightness. Resonant and non-resonant excitation is highlighted by black and red with entangled SPE in light blue, respectively.

an SPE deterministically one needs to place a source spatially and spectrally at the antinode of a cavity mode. The main obstacle in achieving maximum coupling strength is the misalignment between the QD and the antinode of the cavity mode. Two main solutions exist. The first is naturally concerned with QD growth. Deterministic in-situ growth methods allow one to pre-select emitters either in inverted QD chains [87,92] or by optimized droplet-etching [93] within the intended emission band with an accuracy of about $1 \mathrm{meV}$. Thus, an additional spectral fine-tuning is needed by means of temperature tuning [94], varying electric fields [95] or strain-tuning [78]. Another solution is to scan and register the dot. The original idea was to mark the position of single QD and apply multiple digital etching steps [96,97]. An all-optical method was adopted to minimize the lithography steps [98]. One promising class of photonic crystal cavity design is the modulated mode-gap cavity [99]. One way of achieving such a cavity is to deposit a low refractive index material on top of a photonic crystal waveguide as shown in Fig 4e, f. The small photonic mode volume guarantees high Q cavities using an optical system, and thus greatly increases the probability of successful coupling between a QD and a cavity mode [86]. The lithography steps are straightforward, with a spatial precision of $\pm 25 \mathrm{~nm}$ making the techniqure promising for scalable on-chip SPE systems [99].

\subsection{III-Nitrides}

Single photon emission can be realized in the III-nitride system with the formation of QDs making use of the 3-dimensional barriers that can be created by contrasting the potentials of different nitride alloys such as AlN, AlGaN, $\mathrm{GaN}$, InGaN, and InN. Due to the extensive use of GaN in the lighting and diode laser industry, its associated fabrication techniques have thus advanced at a tremendous rate. This mass adoption of LED lighting has seen the price of the technology benefit from the economies of scale. In terms of the industrial maturity for large scale production, nitride technology is more readily scalable compared to other single photon emitters using exotic materials and techniques such as single trapped atoms [100], diamond nitrogen vacancy centers [101], and 2D semiconductors [21]. Despite the achievements in the past decades on Arsenide-based QD single photon emitters in the infrared, wider bandgap 


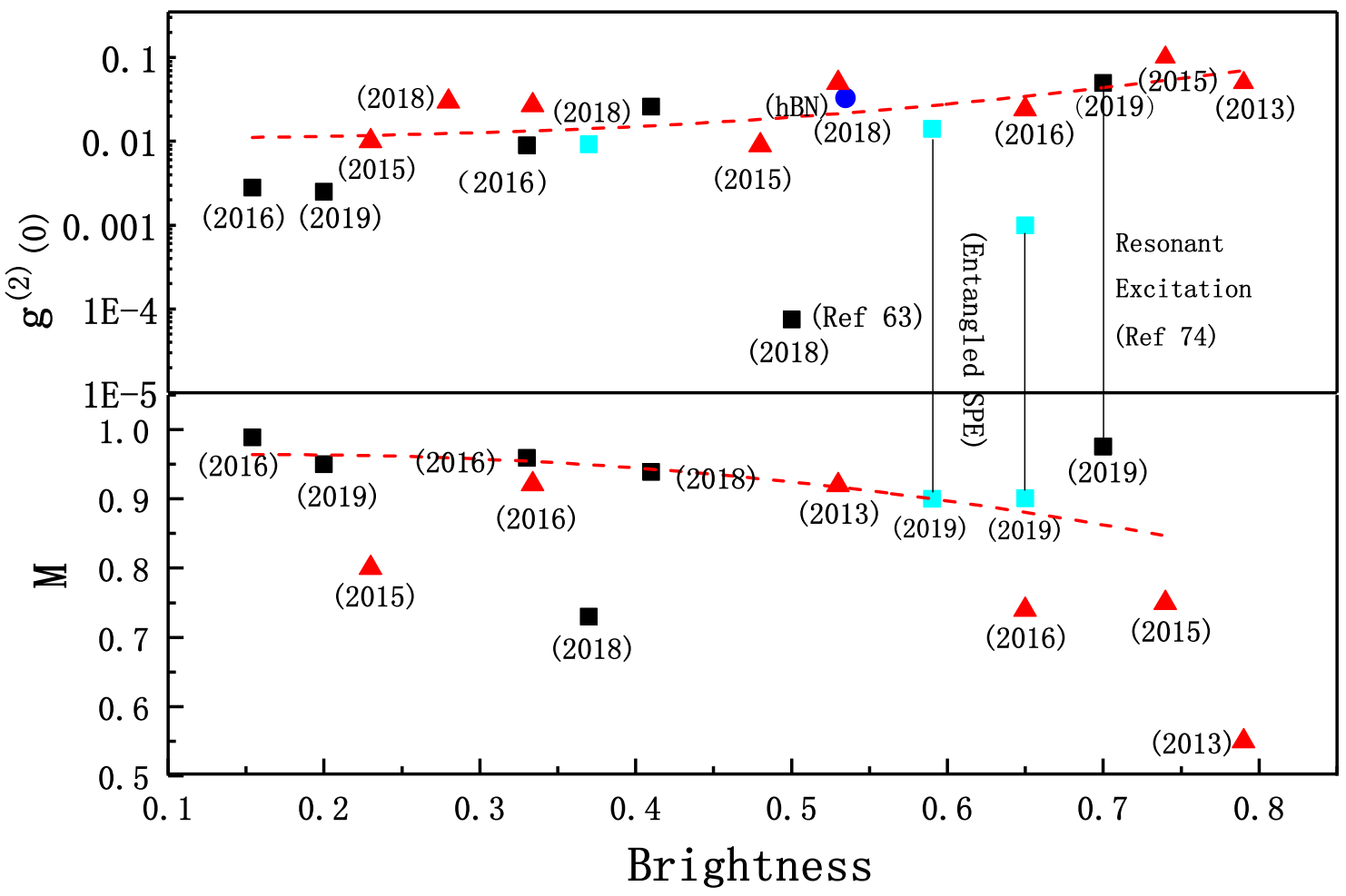

图 6: Purity and indistinguishability as a function of brightness summarized from Table1 with a trend indicated by red-dotted lines. Red triangles are non-resonant excitation while black squares are SPEs with resonant excitation. The blue circle is from $\mathrm{hBN}$ and the light blue squares are photon-pair SPEs.

materials remain desireable due to large availability of fast, and low-cost single photon detectors which can work at room temperature.

Scalability aside, there are a number of major advantages of nitride based single photon emitters, namely temperature stability, polarisability, and wide tuneability. III-Nitride materials have been reported to give single photon emission at room temperature owing to the wide band offsets which are available between different nitride alloys, preventing carrier escape from a QD. The tuneablity of emission ranges from the UV down to the red side of the visible electromagnetic spectrum via bandgap engineering and QD size control. Typically UV single photons are produced by GaN QDs in AlGaN or AlN with photon energies up to 4.35 eV [102]. InGaN in GaN QDs can emit single photons from blue range [103] down to $2.0 \mathrm{eV} \mathrm{[104]} \mathrm{in} \mathrm{the} \mathrm{red.} \mathrm{In} \mathrm{addition,} \mathrm{fine} \mathrm{tuning} \mathrm{of} \mathrm{the} \mathrm{QD} \mathrm{emission} \mathrm{energy} \mathrm{can}$ also be achieved through applying external bias countering the large built-in piezoelectric field in GaN crystals [105]. Moreover, nitride QDs have been shown to emit polarised light [106,107], important for applications in quantum key distribution such as the BB84 scheme [108].

\subsubsection{Material properties}

The majority of III-nitride emitters studied exhibit Wurtzite symmetry, which is non-centrosymmetric. The P63mc space group gives rise to a strong piezoelectric field when the crystal is under compressive or tensile strain. Lattice mismatch between the lower bandgap QD material and the higher bandgap barrier material causes large peizoelectric fields in the QD, giving rise to the quantum confined Stark effect, polarising the electrons and holes. As a consequence, there is reduced electron-hole wavefunction overlap which substantially decreases the radiative recombination rate of the system. The long emission lifetimes of a single photon emitter limits the speed at which these single photons can be generated. For extreme cases, lifetimes in excess of $1 \mu$ s [109] has been seen. Successful growth of QDs in the non-polar plane can effectively reduce the internal field with lifetimes reduced by an order of 
magnitude [39, 110].

\subsubsection{Single photons from GaN based dots}

There are many schemes of growing QDs in the nitride systems. The first nitride single photon emitter was grown in the SK growth mode with MOVPE demonstrated by Kako et al. [111]. Single quantum dot emission was observed at $3.5 \mathrm{eV}$ and an antibunching dip was seen with a $g^{(2)}(0)$ of 0.42 . In the visible wavelength range, single photon emission was acheived with InGaN QDs in GaN grown by MOVPE and using droplet epitaxy [103]. The InGaN QD at $2.8 \mathrm{eV}$ meant that two-photon excitation from a common $800 \mathrm{~nm}$ Ti:Saph laser can be used to selectively excite the QD with higher selectivity and reduced background. This led to a measured $g^{(2)}(0)$ of less than 0.3 . Recently, a $g^{(2)}(0)$ of 0.02 was seen in a GaN QD system formed at the macrostep edges of a GaN/AlGaN quantum well grown in MOCVD [112]. This unprecedented value in nitride systems is a remarkable breakthrough, however these values are not comparable to arsenide systems where $g^{(2)}(0)$ values up to 3 orders of magnitude lower have been achieved.

\subsubsection{Temperature stability}

The large band offsets and strong carrier confinement nature of III-nitride QDs enables large potential barriers to be formed to prevent carrier escape from a QD. Very early on, Kako et al. had already highlighted this by the successful demonstration of single photon emission with a $g^{(2)}(0)$ of 0.53 at $200 \mathrm{~K}$ [111]. This early achievement showed the practicality of nitride semiconductors for the generation of single photons at Peltier-reachable temperatures. In 2014, Holmes et al. [102] used MOVPE to grow GaN nanowires defined by $25 \mathrm{~nm}$ apertures. The resulting nanowires had a $\mathrm{GaN}$ QD formed at the tip surrounded by an AlGaN capping layer. The small QD had emission in the UV at $4.35 \mathrm{eV}$ and a $g^{(2)}(0)$ of 0.33 at room temperature. The good temperature performance of this device arises from three factors. (i) A reduction in size which increases the wavefunction overlap, and hence emission probability. (ii) A large band offset vs $k T$, and (iii) a site-controlled approach means that a better background isolation can be obtained. Holmes et al. 2016 went on to report single photon emission at a record $350 \mathrm{~K}$ from nitride systems [113] using a similar approach. The above examples are from GaN QDs formed in AlGaN with relatively high band offsets. GaN crystal localized defects have proved to be able to exhibit high photon purity $(\sim 0.05)$ at a high brightness of $\sim 1.5 \mathrm{MHz}$ at room temperature [114]. The large band offset caused by the high indium incorporation is key in achieving hightemperature single photon operation. With lower indium content blue emitters, temperature stability had been observed by growing the QDs on non-polar crystal planes. It has been shown that by decreasing the internal field, carrier overlap improvement would lead to a faster carrier lifetime in non-polar InGaN/GaN quantum dots [110] [115]. Faster radiative recombination reduces the probability of non-radiative recombination which are activated at higher temperatures. Wang et al. reported blue single photon source from non-polar InGaN system at $220 \mathrm{~K}$ with a $g^{(2)}(0)$ of 0.47 [116].

\subsubsection{Polarisability}

Nitride QDs have regularly been reported to give a linearly polarised emission [117-119]. In quantum key distribution, it is required that information is encoded in the quantum state of the single photons, which is represented by their polarization. Therefore, an ideal single photon emitter for quantum computing and key distribution applications should be capable of generating on-demand single photons with a pre-defined polarization. The direction of linear polarization emission is determined by the anisotropy of strain and shape of the QDs citeBardoux2008. Manipulation of the photon polarization in III-nitride QDs have been achieved using few different methods: A bottom-up approach by controlling the shape of the site of the QD growth [120], a top-down approach by defining the QD shape via lithography [121], and by growing non-polar dots and using the crystal $c$-axis to define the polarization direction [107].

Lundskog et al. demonstrated precise site control of QD emission polarization by growing InGaN QDs on top of hexagonal GaN pyramids in which the apex were elongated [120]. The photon polarization was shown to be defined by the elongation direction, and an average degree of linear polarization of 0.84 was achieved in the 6 different orientations of the pyramids. The bottom down approach for controlling polarization was demonstrated in InGaN/GaN 


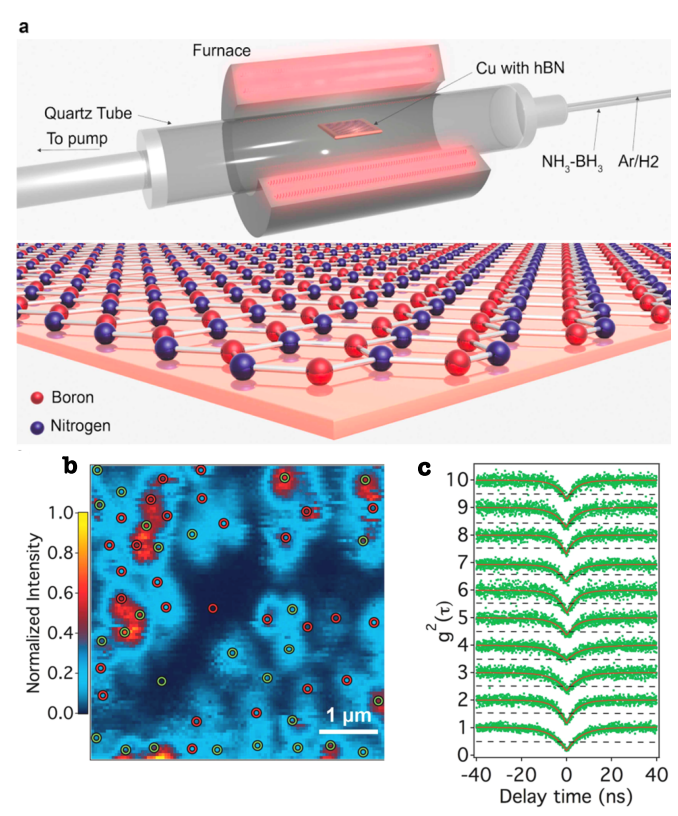

图 7: (a) Schematics of a LPCVD setup to produce hBN film where ammonia borane is used as a CVD precursor. (b) A confocal PL map showing hBN luminescence. (c) hBN single photon measurement with $g^{2}(0)$ within 0.5 from [123].

QDs in nanopillars by Teng et al. [121]. Nanopillars containing a single InGaN quantum disk were etched to create elliptical InGaN QDs which showed a single photon emission with a $g^{(2)}(0)$ of 0.26 , and a degree of linear polarization, up to $P=0.98$ when tuning the aspect ratio of the QD. Deterministic single photon polarization has been shown also in non-polar InGaN QDs. With the origin of the polarization arising from the anisotropy of the strain in the growth plane [116]. Blue emitting non-polar InGaN QDs was shown to emit with a degree of linear polarisation $P=0.84$ [39] in addition to an impressive single photon emission temperature stability of up to $220 \mathrm{~K}$ and a $260 \mathrm{ps}$ lifetime. Moreover, electrically driven emission of polarised single photons has also been observed in such nonpolar systems [122], demonstrating a promising step towards generating on demand high-rate single photons with pre-defined polarization.

\section{3. hBN-base SPEs}

$\mathrm{hBN}$, which is a layered semiconductor with a wide band gap of $5.5 \mathrm{eV}$, has also been reported to be a single photon source [124,125]. It has attracted widespread attention for its capability to enhance electronic and optical properties of 2D material heterostructures [126], and for its natural hyperbolic properties [127]. To date, single photons generated from 2D materials have a broad distribution of lifetime-bandwidth products at room temperature. Defects on hBN generally have a sub-nanoseconds lifetime and are formed from either ion irradiation, chemical etching, or plasma etching [123]. The reason why the single photon emitter can be made in the hBN is mainly due to crystallographic defects $[128,129]$ but the detailed mechanisms are still under debate. Therefore, these emitters usually have problems of uneven spectral distribution, multi-photon emission and low single photon purity, etc. A tuneable ultrabright room temperature single photon source has been generated by the strain control of emission wavelength and the method of greatly reducing photon emission probability [130,131].Through cavity alignment, a high quantum efficiency of radiation transition led to a large mechanical quality factor of 1100 for a $23 \mathrm{MHz}$ mode, at room temperature in high vacuum [37]. In addition, to characterize precisely intrinsic defects hosted in the hBN crystal is still essential. And There is still a long way to go to improve the stability and brightness of single-photon emitters [132].

\section{Conclusions and outlook}

In this paper, we have reviewed the various aspects of III-V compound SPEs. concentrating on III-arsenides, III-nitrides and hBN based SPEs, InGaAs QDs based SPEs have already achieved high purity, high brightness and 
high indistinguishability, simultaneously, and even recently high fidelity for photon-pair generation. Great progress has also been made towards the development of III-nitrides as single photon emitters at elevated temperatures and also ones based on hBN in recent years. This has arisen from collaborative research of many groups spread out across the world. Advances have been made in particular with respect to high temperature operation and single photon emission covering the main communication spectrum from $800 \mathrm{~nm}$ to $1550 \mathrm{~nm}$ [75]. However, there is still much work to be done in terms of both theoretical and experimental fields.

Scalability is the next major criterion for the development of SPEs, with the key performance metrics of brightness, indistinguishability and purity have errors below $1 \%$ or so. in particularly one may consider chip-based photonics as it offers crucial advantages in terms of stability and portability [133]. Ideal single photon emitters will provide all-optical processing ability armed with many-photon entangled states and light-matter interaction. For this to be realized, enhancing coupling quality, electron spin resonance and ion implantation studies are needed [134].

We spent most of the paper discuss about single photons from one source. However perspective quantum repeater scenarios process single photon interference from remote quantum nodes [135]. In this respect, one of the next challenge is to realize high efficiency two-photon interference. Inhibiting spectral diffusion and resonant excitation greatly improves the indistinguishability and visibility of two remote sources [136], and we expect more bright remote sources will be developed exhibiting many-photon interference, thus enabling applications such as boson sampling measurements [137,138] and quantum self-organization [139] to be undertaken.

The crystallographic and electronic structure and origin of the hBN defect is still under debate. Much more effort is required to improve the growth of III-nitrides and 2D materials, particularly in attempts to control identical QDs and spectrally stable defects [140]. High temperature SPEs are of significant importance for practical operations [141]. Expertise gained developing III-arsenides sources can be used to improve nitrides SPEs and help overcome decoherence issues. Electrically-driven devices and hBN transfer techniques also promise high-performance SPE devices, spurring further studies of single photon sources and single electron and multi-particle interactions.

\section{参考文献}

[1] Santori C, Fattal D, Yamamoto Y, Single-photon devices and applications (Wiley, Germany, 2010)

[2] Hennessy K, Badolato A, Winger M, Gerace D, Atatüre M, Gulde S, Fält S, Hu EL, Imamoğlu A, Quantum nature of a strongly coupled single quantum dot-cavity system. Nature 445(7130), 896 (2007). DOI 10.1038/ nature05586. URL http://dx.doi.org/10.1038/nature05586

[3] Harrow AW, Montanaro A, Quantum computational supremacy. Nature 549, 203 (2017). URL https:// doi.org/10.1038/nature23458

[4] Hu L, Wu SH, Cai W, Ma Y, Mu X, Xu Y, Wang H, Song Y, Deng DL, Zou CL, Sun L, Quantum generative adversarial learning in a superconducting quantum circuit. Science Advances 5(1) (2019)

[5] Qiang X, Zhou X, Wang J, Wilkes CM, Loke T, O'Gara S, Kling L, Marshall GD, Santagati R, Ralph TC, et al., Large-scale silicon quantum photonics implementing arbitrary two-qubit processing. Nat. Photonics 12(9), 534 (2018). DOI 10.1038/s41566-018-0236-y. URL http://dx.doi.org/10.1038/ s41566-018-0236-y

[6] Kok P, Munro WJ, Nemoto K, Ralph TC, Dowling JP, Milburn GJ, Linear optical quantum computing with photonic qubits. Rev. Mod. Phys. 79, 135 (2007). DOI 10.1103/RevModPhys.79.135. URL https : / / ink . aps.org/doi/10.1103/RevModPhys.79.135

[7] Giovannetti V, Lloyd S, Maccone L, Advances in quantum metrology. Nature Photonics 5(4), 222 (2011). DOI 10.1038/nphoton.2011.35. URL http://dx.doi.org/10.1038/nphoton.2011.35

[8] Chen MC, Liu C, Luo YH, Huang HL, Wang BY, Wang XL, Li L, Liu NL, Lu CY, Pan JW, Experimental demonstration of quantum pigeonhole paradox. PNAS; Proceedings of the National Academy of Sciences 116(5), 1549 (2019) 
[9] Liao SK, Cai WQ, Handsteiner J, et al., Satellite-relayed intercontinental quantum network. Phys. Rev. Lett. 120, 030501 (2018). DOI 10.1103/PhysRevLett.120.030501. URL https://Iink.aps.org/doi/10. 1103 /PhysRevLett.120.030501

[10] Kuhn A, Hennrich M, Rempe G, Deterministic single-photon source for distributed quantum networking. Phys. Rev. Lett. 89, 067901 (2002). DOI 10.1103/PhysRevLett.89.067901. URL https:// link.aps.org/ doi/10.1103/PhysRevLett.89.067901

[11] Chu S, Cold atoms and quantum control. Nature 416(6877), 206 (2002). DOI 10.1038/416206a. URL http: //dx.doi.org/10.1038/416206a

[12] Haroche S, Kleppner D, Cavity quantum electrodynamics. Phys. Today 42(1), 24 (1989). DOI 10.1063/1. 881201. URL http://dx.doi.org/10.1063/1.881201

[13] Dietsche EK, Larrouy A, Haroche S, Raimond JM, Brune M, Gleyzes S, High-sensitivity magnetometry with a single atom in a superposition of two circular rydberg states. Nat. Phys. 15(4), 326 (2019). DOI 10.1038/ s41567-018-0405-4. URL http://dx.doi.org/10.1038/s41567-018-0405-4

[14] Schmidt PO, Rosenband T, Langer C, Itano WM, Bergquist JC, Wineland DJ, Spectroscopy using quantum logic. Science 309(5735), 749 (2005)

[15] Almendros M, Huwer J, Piro N, Rohde F, Schuck C, Hennrich M, Dubin F, Eschner J, Bandwidthtunable single-photon source in an ion-trap quantum network. Phys. Rev. Lett. 103, 213601 (2009). DOI 10.1103/PhysRevLett.103.213601. URL https://link.aps.org/doi/10.1103/PhysRevLett. 103.213601

[16] Higginbottom DB, Slodička L, Araneda G, Lachman L, Filip R, Hennrich M, Blatt R, Pure single photons from a trapped atom source. New J. Phys. 18(9), 093038 (2016). DOI 10.1088/1367-2630/18/9/093038. URL http://dx.doi.org/10.1088/1367-2630/18/9/093038

[17] Senellart P, Solomon G, White A, High-performance semiconductor quantum-dot single-photon sources. Nat. Nanotechnol. 12, 1026 (2017)

[18] Benedikter J, Kaupp H, Hümmer T, Liang Y, Bommer A, Becher C, Krueger A, Smith JM, Hänsch TW, Hunger D, Cavity-enhanced single-photon source based on the silicon-vacancy center in diamond. Phys. Rev. Applied 7, 024031 (2017). DOI 10.1103/PhysRevApplied.7.024031. URL https://Iink.aps.org/doi/10 . 1103 /PhysRevApplied. 7.024031

[19] Lounis B, Moerner WE, Single photons on demand from a single molecule at room temperature. Nature 407(6803), 491 (2000). DOI 10.1038/35035032. URL http://dx.doi.org/10 . 1038/35035032

[20] Tran TT, Bray K, Ford MJ, Toth M, Aharonovich I, Quantum emission from hexagonal boron nitride monolayers. Nat. Nanotechnol. 11(1), 37 (2015). DOI 10.1038/nnano.2015.242. URL http: / / dx.doi.org/10. 1038 /nnano.2015.242

[21] He YM, Clark G, Schaibley JR, He Y, Chen MC, Wei YJ, Ding X, Zhang Q, Yao W, Xu X, Lu CY, Pan JW, Single quantum emitters in monolayer semiconductors. Nat. Nanotechnol. 10(6), 497 (2015). DOI 10.1038/ nnano.2015.75. URL http://dx.doi.org/10.1038/nnano.2015.75

[22] Chakraborty C, Kinnischtzke L, Goodfellow KM, Beams R, Vamivakas AN, Voltage-controlled quantum light from an atomically thin semiconductor. Nat. Nanotechnol. 10(6), 507 (2015). DOI 10.1038/nnano.2015.79. URL http://dx.doi.org/10.1038/nnano.2015.79 
[23] Wang X, Alexander-Webber JA, Jia W, Reid BPL, Stranks SD, Holmes MJ, Chan CCS, Deng C, Nicholas RJ, Taylor RA, Quantum dot-like excitonic behavior in individual single walled-carbon nanotubes. Sci. Rep. 6(1) (2016). DOI 10.1038/srep37167. URL http://dx.doi.org/10.1038/srep37167

[24] He X, Htoon H, Doorn SK, Pernice WHP, Pyatkov F, Krupke R, Jeantet A, Chassagneux Y, Voisin C, Carbon nanotubes as emerging quantum-light sources. Nat. Mater. 17(8), 663 (2018). DOI 10.1038/ s41563-018-0109-2. URL http://dx.doi.org/10.1038/s41563-018-0109-2

[25] Ding X, He Y, Duan ZC, Gregersen N, Chen MC, Unsleber S, Maier S, Schneider C, Kamp M, Höfling S, Lu CY, Pan JW, On-demand single photons with high extraction efficiency and near-unity indistinguishability from a resonantly driven quantum dot in a micropillar. Phys. Rev. Lett. 116, 020401 (2016). DOI 10.1103/PhysRevLett.116.020401. URL https://link.aps.org/doi/10.1103/PhysRevLett. 116.020401

[26] Somaschi N, Giesz V, De Santis L, Loredo JC, Almeida MP, Hornecker G, Portalupi SL, Grange T, Antón C, Demory J, Gómez C, Sagnes I, Lanzillotti-Kimura ND, Lemaítre A, Auffeves A, White AG, Lanco L, Senellart P, Near-optimal single-photon sources in the solid state. Nat. Photonics 10, 340 EP (2016). URL https://doi.org/10.1038/nphoton.2016.23

[27] Gammon D, Snow ES, Shanabrook BV, Katzer DS, Park D, Homogeneous linewidths in the optical spectrum of a single gallium arsenide quantum dot. Science 273(5271), 87 (1996)

[28] Rice JH, Robinson JW, Na JH, Lee KH, Taylor RA, Williams DP, O'Reilly EP, Andreev AD, Arakawa Y, Yasin $\mathrm{S}$, Biexciton and exciton dynamics in single ingan quantum dots. Nanotechnology 16(9), 1477 (2005). DOI 10.1088/0957-4484/16/9/010. URL http://dx.doi.org/10.1088/0957-4484/16/9/010

[29] Shields AJ, Semiconductor quantum light sources. Nat. Photonics 1(4), 215 (2007). DOI 10.1038/nphoton. 2007.46. URL http://dx.doi.org/10.1038/nphoton.2007.46

[30] Andreev AD, O'Reilly EP, Optical transitions and radiative lifetime in gan/aln self-organized quantum dots. Appl. Phys. Lett. 79(4), 521 (2001). DOI 10.1063/1.1386405. URL http: / / dx . doi .org/10.1063/1. 1386405

[31] Lee KH, Brossard FSF, Hadjipanayi M, Xu X, Waldermann F, Green AM, Sharp DN, Turberfield AJ, Williams DA, Taylor RA, Towards registered single quantum dot photonic devices. Nanotechnology 19(45), 455307 (2008). DOI 10.1088/0957-4484/19/45/455307. URL http://dx.doi.org/10.1088/0957-4484/ $19 / 45 / 455307$

[32] Schöll E, Hanschke L, Schweickert L, Zeuner KD, Reindl M, Covre da Silva SF, Lettner T, Trotta R, Finley JJ, Müller K, et al., Resonance fluorescence of gaas quantum dots with near-unity photon indistinguishability. Nano Lett. 19(4), 2404 (2019). DOI 10.1021/acs.nanolett.8b05132. URL http://dx.doi.org/10. 1021 /acs.nanolett.8b05132

[33] Miyazawa T, Takemoto K, Sakuma Y, Hirose S, Usuki T, Yokoyama N, Takatsu M, Arakawa Y, Single-photon generation in the 1.55-ţm optical-fiber band from an inas/inp quantum dot. Japanese Journal of Applied Physics 44(No. 20), L620 (2005). DOI 10.1143/jjap.44.1620. URL http: / / dx. doi . org/ 10 . 1143 / J JAP . 44. $\mathrm{L} 620$

[34] Fotue AJ, Kenfack SC, Issofa N, Tiotsop M, Fotsin H, Mainimo E, Fai LC, Energy levels of magneto-optical polaron in spherical quantum dot - part 1: Strong coupling. J. Semicond. 36(9), 092001 (2015). DOI 10.1088/ 1674-4926/36/9/092001. URL http://dx.doi.org/10.1088/1674-4926/36/9/092001 
[35] Michler P, Imamoğlu A, Mason MD, Carson PJ, Strouse GF, Buratto SK, Quantum correlation among photons from a single quantum dot at room temperature. Nature 406(6799), 968 (2000). DOI 10.1038/35023100. URL http://dx.doi.org/10.1038/35023100

[36] Mahler B, Spinicelli P, Buil S, Quelin X, Hermier JP, Dubertret B, Towards non-blinking colloidal quantum dots. Nat. Mater. 7(8), 659 (2008). DOI 10.1038/nmat2222. URL http://dx.doi.org/10.1038/ nmat 2222

[37] Shandilya PK, Fröch JE, Mitchell M, Lake DP, Kim S, Toth M, Behera B, Healey C, Aharonovich I, Barclay PE, Hexagonal boron nitride cavity optomechanics. Nano Lett. 19(2), 1343 (2019). DOI 10.1021/acs.nanolett. 8b04956. URL http://dx.doi.org/10.1021/acs.nanolett.8b04956

[38] Brown RH, Twiss RQ, Interferometry of the Intensity Fluctuations in Light. I. Basic Theory: The Correlation between Photons in Coherent Beams of Radiation. Proc. R. Soc. London, Ser. A 242, 300 (1957). DOI 10.1098/rspa.1957.0177

[39] Puchtler TJ, Wang T, Ren CX, Tang F, Oliver RA, Taylor RA, Zhu T, Ultrafast, Polarized, Single-Photon Emission from m-Plane InGaN Quantum Dots on GaN Nanowires. Nano Lett. 16(12), 7779 (2016). DOI 10.1021/acs.nanolett.6b03980

[40] Giesz V, Gazzano O, Nowak AK, Portalupi SL, Lemaitre A, Sagnes I, Lanco L, Senellart P, Influence of the purcell effect on the purity of bright single photon sources. Appl. Phys. Lett. 103(3), 033113 (2013). DOI 10.1063/1.4813902. URL http://dx.doi.org/10.1063/1.4813902

[41] Flagg EB, Polyakov SV, Thomay T, Solomon GS, Dynamics of nonclassical light from a single solid-state quantum emitter. Phys. Rev. Lett. 109, 163601 (2012). DOI 10.1103/PhysRevLett.109.163601. URL https: //link.aps.org/doi/10.1103/PhysRevLett.109.163601

[42] Fischer KA, Müller K, Lagoudakis KG, Vučković J, Dynamical modeling of pulsed two-photon interference. New J. Phys. 18(11), 113053 (2016). DOI 10.1088/1367-2630/18/11/113053. URL http: / / dx . doi . org/ $10.1088 / 1367-2630 / 18 / 11 / 113053$

[43] Hong CK, Ou ZY, Mandel L, Measurement of subpicosecond time intervals between two photons by interference. Phys. Rev. Lett. 59, 2044 (1987). DOI 10.1103/PhysRevLett.59.2044. URL https:// link.aps. org/doi/10.1103/PhysRevLett.59.2044

[44] Wang XL, Cai XD, Su ZE, Chen MC, Wu D, Li L, Liu NL, Lu CY, Pan JW, Quantum teleportation of multiple degrees of freedom of a single photon. Nature 518(7540), 516 (2015). DOI 10.1038/nature14246. URL http: //dx.doi.org/10.1038/nature14246

[45] Aaronson S, Arkhipov A, The computational complexity of linear optics. Theory of Computing 9(1), 143 (2013). DOI 10.4086/toc.2013.v009a004. URL http://dx.doi.org/10.4086/toc.2013. v009a004

[46] Michler P, Quantum Dots for Quantum Information Technologies. Nano-Optics and Nanophotonics (Springer International Publishing, Gewerbestrasse 11, 6330 Cham, Switzerland, 2017). DOI 10.1007/ 978-3-319-56378-7. URL http://dx.doi.org/10.1007/978-3-319-56378-7

[47] Liu F, Brash AJ, O'Hara J, Martins LMPP, Phillips CL, Coles RJ, Royall B, Clarke E, Bentham C, Prtljaga $\mathrm{N}$, et al., High purcell factor generation of indistinguishable on-chip single photons. Nat. Nanotechnol. 13(9), 835 (2018). DOI 10.1038/s41565-018-0188-x. URL http://dx.doi.org/10.1038/ s41565-018-0188-x 
[48] Munsch M, Malik NS, Dupuy E, Delga A, Bleuse J, Gérard JM, Claudon J, Gregersen N, Mørk J, Dielectric gaas antenna ensuring an efficient broadband coupling between an inas quantum dot and a gaussian optical beam. Phys. Rev. Lett. 110, 177402 (2013). DOI 10.1103/PhysRevLett.110.177402. URL https : / I ink . aps.org/doi/10.1103/PhysRevLett.110.177402

[49] Claudon J, Bleuse J, Malik NS, Bazin M, Jaffrennou P, Gregersen N, Sauvan C, Lalanne P, Gérard JM, A highly efficient single-photon source based on a quantum dot in a photonic nanowire. Nat. Photonics 4(3), 174 (2010). DOI 10.1038/nphoton.2009.287x. URL http://dx.doi.org/10.1038/nphoton.2009.287x

[50] Gschrey M, Thoma A, Schnauber P, Seifried M, Schmidt R, Wohlfeil B, Krüger L, Schulze JH, Heindel T, Burger S, et al., Highly indistinguishable photons from deterministic quantum-dot microlenses utilizing threedimensional in situ electron-beam lithography. Nat. Commun. 6(1) (2015). DOI 10.1038/ncomms8662. URL http://dx.doi.org/10.1038/ncomms 8662

[51] Sapienza L, Davanço M, Badolato A, Srinivasan K, Nanoscale optical positioning of single quantum dots for bright and pure single-photon emission. Nat. Commun. 6(1) (2015). DOI 10.1038/ncomms8833. URL http://dx.doi.org/10.1038/ncomms 8833

[52] Gazzano O, Michaelis de Vasconcellos S, Arnold C, Nowak A, Galopin E, Sagnes I, Lanco L, Lemaître A, Senellart P, Bright solid-state sources of indistinguishable single photons. Nat. Commun. 4(1) (2013). DOI 10.1038/ncomms2434. URL http://dx.doi .org/10.1038/ncomms2434

[53] Unsleber S, He YM, Gerhardt S, Maier S, Lu CY, Pan JW, Gregersen N, Kamp M, Schneider C, Höfling S, Highly indistinguishable on-demand resonance fluorescence photons from a deterministic quantum dot micropillar device with 74\% extraction efficiency. Opt. Express 24(8), 8539 (2016). DOI 10.1364/oe.24.008539. URL http://dx.doi.org/10.1364/OE.24.008539

[54] Einstein A, Podolsky B, Rosen N, Can quantum-mechanical description of physical reality be considered complete? Phys. Rev. 47, 777 (1935). DOI 10.1103/PhysRev.47.777. URL https: / / link.aps.org/doi/ $10.1103 /$ PhysRev.47.777

[55] Briegel HJ, Dür W, Cirac JI, Zoller P, Quantum repeaters: The role of imperfect local operations in quantum communication. Phys. Rev. Lett. 81, 5932 (1998). DOI 10.1103/PhysRevLett.81.5932. URL https:// link.aps.org/doi/10.1103/PhysRevLett.81.5932

[56] Bennett CH, Brassard G, Crépeau C, Jozsa R, Peres A, Wootters WK, Teleporting an unknown quantum state via dual classical and einstein-podolsky-rosen channels. Phys. Rev. Lett. 70, 1895 (1993). DOI 10.1103/ PhysRevLett.70.1895. URL https: / / ink.aps.org/doi/10.1103/PhysRevLett. 70.1895

[57] Raussendorf R, Briegel HJ, A one-way quantum computer. Phys. Rev. Lett. 86, 5188 (2001). DOI 10.1103/ PhysRevLett.86.5188. URL https:// link.aps.org/doi/10.1103/PhysRevLett.86.5188

[58] Andersen UL, Ralph TC, High-fidelity teleportation of continuous-variable quantum states using delocalized single photons. Phys. Rev. Lett. 111, 050504 (2013). DOI 10.1103/PhysRevLett.111.050504. URL https: //link.aps.org/doi/10.1103/PhysRevLett.111.050504

[59] Goldstein L, Glas F, Marzin JY, Charasse MN, Le Roux G, Growth by molecular beam epitaxy and characterization of inas/gaas strainedlayer superlattices. Appl. Phys. Lett. 47(10), 1099 (1985). DOI 10.1063/1.96342. URL http://dx.doi.org/10.1063/1.96342

[60] Clarke E, Spencer P, Harbord E, Howe P, Murray R, Growth, optical properties and device characterisation of inas/gaas quantum dot bilayers. J. Phys. Conf. Ser. 107, 012003 (2008). DOI 10.1088/1742-6596/107/1/ 012003. URL http://dx.doi.org/10.1088/1742-6596/107/1/012003 
[61] Konishi T, Clarke E, Burrows CW, Bomphrey JJ, Murray R, Bell GR, Spatial regularity of inas-gaas quantum dots: quantifying the dependence of lateral ordering on growth rate. Sci. Rep. 7(1) (2017). DOI 10.1038/ srep42606. URL http://dx.doi.org/10.1038/srep42606

[62] Haffouz S, Zeuner KD, Dalacu D, Poole PJ, Lapointe J, Poitras D, Mnaymneh K, Wu X, Couillard M, Korkusinski M, et al., Bright single inasp quantum dots at telecom wavelengths in position-controlled inp nanowires: The role of the photonic waveguide. Nano Lett. 18(5), 3047 (2018). DOI 10.1021/acs.nanolett.8b00550. URL http://dx.doi.org/10.1021/acs.nanolett.8b00550

[63] Schweickert L, Jöns KD, Zeuner KD, Covre da Silva SF, Huang H, Lettner T, Reindl M, Zichi J, Trotta R, Rastelli A, et al., On-demand generation of background-free single photons from a solid-state source. Appl. Phys. Lett. 112(9), 093106 (2018). DOI 10.1063/1.5020038. URL http://dx.doi.org/10.1063/1. 5020038

[64] Huber D, Reindl M, Huo Y, Huang H, Wildmann JS, Schmidt OG, Rastelli A, Trotta R, Highly indistinguishable and strongly entangled photons from symmetric gaas quantum dots. Nature Communications 8, 15506 (2017). DOI 10.1038/ncomms15506. URL http://dx.doi.org/10.1038/ncomms 15506

[65] Patton B, Langbein W, Woggon U, Trion, biexciton, and exciton dynamics in single self-assembled cdse quantum dots. Phys. Rev. B 68, 125316 (2003). DOI 10.1103/PhysRevB.68.125316. URL https : / / link . aps . org/doi/10.1103/PhysRevB.68.125316

[66] Portalupi SL, Hornecker G, Giesz V, Grange T, Lemaître A, Demory J, Sagnes I, Lanzillotti-Kimura ND, Lanco L, Auffèves A, et al., Bright phonon-tuned single-photon source. Nano Lett. 15(10), 6290 (2015). DOI 10.1021/acs.nanolett.5b00876. URL http://dx.doi.org/10.1021/acs.nanolett.5b00876

[67] Santori C, Fattal D, Vuckovic J, Solomon GS, Yamamoto Y, Single-photon generation with inas quantum dots. New J. Phys. 6, 89 (2004). DOI 10.1088/1367-2630/6/1/089. URL http://dx.doi.org/10.1088/ $1367-2630 / 6 / 1 / 089$

[68] Marzin JY, Photoluminescence of single inas quantum dots obtained by self-organized growth on gaas. Phys. Rev. Lett. 73(5), 716 (1994). DOI 10.1103/PhysRevLett.73.716

[69] Fafard S, Leonard D, Merz JL, Petroff PM, Selective excitation of the photoluminescence and the energy levels of ultrasmall ingaas/gaas quantum dots. Appl. Phys. Lett. 65(11), 1388 (1994). DOI 10.1063/1.112060. URL http://dx.doi.org/10.1063/1.112060

[70] Michler P, Kiraz A, Becher C, Schoenfeld WV, Petroff PM, Zhang L, Hu E, Imamoglu A, A quantum dot single-photon turnstile device. Science 290(5500), 2282 (2000)

[71] Benisty H, De Neve H, Weisbuch C, Impact of planar microcavity effects on light extraction-part ii: selected exact simulations and role of photon recycling. IEEE J. Quantum Electron. 34(9), 1632 (1998)

[72] Giesz V, Portalupi SL, Grange T, Antón C, De Santis L, Demory J, Somaschi N, Sagnes I, Lemaître A, Lanco L, Auffèves A, Senellart P, Cavity-enhanced two-photon interference using remote quantum dot sources. Phys. Rev. B 92, 161302 (2015). DOI 10.1103/PhysRevB.92.161302. URL https:// link.aps.org/doi/ $10.1103 /$ PhysRevB.92.161302

[73] Wang H, Duan ZC, Li YH, Chen S, Li JP, He YM, Chen MC, He Y, Ding X, Peng CZ, Schneider C, Kamp M, Höfling S, Lu CY, Pan JW, Near-transform-limited single photons from an efficient solid-state quantum emitter. Phys. Rev. Lett. 116, 213601 (2016). DOI 10.1103/PhysRevLett.116.213601. URL https : / / ink . aps . org/doi/10.1103/PhysRevLett.116.213601 
[74] He YM, Wang H, Gerhardt S, Winkler K, Jurkat J, Polarized indistinguishable single photons from a quantum dot in an elliptical micropillar. arXiv:1809.10992v1 [physics.optics] (2018)

[75] Müller T, Skiba-Szymanska J, Krysa AB, Huwer J, Felle M, Anderson M, Stevenson RM, Heffernan J, Ritchie DA, Shields AJ, A quantum light-emitting diode for the standard telecom window around 1,550nm. Nature Communications 9(1) (2018). DOI 10.1038/s41467-018-03251-7. URL http://dx.doi.org/10 . $1038 /$ s 41467-018-03251-7

[76] Santori C, Fattal D, Vučković J, Solomon GS, Yamamoto Y, Indistinguishable photons from a single-photon device. Nature 419(6907), 594 (2002). DOI 10.1038/nature01086. URL http: / / dx.doi . org/10.1038/ nature 01086

[77] Hanschke L, Fischer KA, Appel S, Lukin D, Wierzbowski J, Sun S, Trivedi R, Vučković J, Finley JJ, Müller K, Quantum dot single-photon sources with ultra-low multi-photon probability. npj Quantum Information 4(1) (2018). DOI 10.1038/s41534-018-0092-0. URL http://dx.doi.org/10.1038/ S41534-018-0092-0

[78] Fischbach S, Kaganskiy A, Tauscher EBY, Gericke F, Thoma A, Schmidt R, Strittmatter A, Heindel T, Rodt S, Reitzenstein S, Efficient single-photon source based on a deterministically fabricated single quantum dot microstructure with backside gold mirror. Appl. Phys. Lett. 111(1), 011106 (2017). DOI 10.1063/1.4991389. URL http://dx.doi.org/10.1063/1.4991389

[79] Tanaka K, Nakamura T, Takamatsu W, Yamanishi M, Lee Y, Ishihara T, Cavity-induced changes of spontaneous emission lifetime in one-dimensional semiconductor microcavities. Phys. Rev. Lett. 74, 3380 (1995). DOI 10.1103/PhysRevLett.74.3380. URL https:// link.aps.org/doi/10.1103/PhysRevLett.74. 3380

[80] Bayer M, Reinecke TL, Weidner F, Larionov A, McDonald A, Forchel A, Inhibition and enhancement of the spontaneous emission of quantum dots in structured microresonators. Phys. Rev. Lett. 86, 3168 (2001). DOI 10.1103/PhysRevLett.86.3168. URL https://link.aps.org/doi/10.1103/PhysRevLett.86. 3168

[81] Peter E, Senellart P, Martrou D, Lemaître A, Hours J, Gérard JM, Bloch J, Exciton-photon strong-coupling regime for a single quantum dot embedded in a microcavity. Phys. Rev. Lett. 95, 067401 (2005). DOI 10. 1103/PhysRevLett.95.067401. URL https://link.aps.org/doi/10.1103/PhysRevLett.95. 067401

[82] Purcell EM, Spontaneous emission probabilities at radio frequencies. NATO ASI Series pp. 839839 (1995). DOI 10.1007/978-1-4615-1963-8_40. URL http://dx.doi.org/10.1007/ 978-1-4615-1963-8_40

[83] Thompson RJ, Turchette QA, Carnal O, Kimble HJ, Nonlinear spectroscopy in the strong-coupling regime of cavity qed. Phys. Rev. A 57, 3084 (1998). DOI 10.1103/PhysRevA.57.3084. URL https: / / link. aps. org/doi/10.1103/PhysRevA.57.3084

[84] Park KD, May MA, Leng H, Wang J, Kropp JA, Gougousi T, Pelton M, Raschke MB, Tip-enhanced strong coupling spectroscopy, imaging, and control of a single quantum emitter. arXiv:1902.10314v1 [physics.optics] (2019)

[85] Wang H, Hu H, Chung TH, Qin J, Yang X, Li JP, Liu RZ, Zhong HS, He YM, Ding X, Deng YH, Dai Q, Huo YH, Höfling S, Lu CY, Pan JW, On-demand semiconductor source of entangled photons which simultaneously has high fidelity, efficiency, and indistinguishability. Phys. Rev. Lett. 122, 113602 (2019). DOI 10.1103/PhysRevLett.122.113602. URL https://Iink.aps.org/doi/10.1103/PhysRevLett. 
[86] Nuttall LP, Brossard FSF, Lennon SA, Reid BPL, Wu J, Griffiths J, Taylor RA, Optical fabrication and characterisation of su-8 disk photonic waveguide heterostructure cavities. Optics Express 25(20), 24615 (2017). DOI 10.1364/oe.25.024615. URL http://dx.doi.org/10.1364/OE.25.024615

[87] Chung TH, Juska G, Moroni ST, Pescaglini A, Gocalinska A, Pelucchi E, Selective carrier injection into patterned arrays of pyramidal quantum dots for entangled photon light-emitting diodes. Nature Photonics 10(12), 782 (2016). DOI 10.1038/nphoton.2016.203. URL http://dx.doi.org/10.1038/nphoton. 2016. 203

[88] Huo YH, Rastelli A, Schmidt OG, Ultra-small excitonic fine structure splitting in highly symmetric quantum dots on gaas (001) substrate. Applied Physics Letters 102(15), 152105 (2013). DOI 10.1063/1.4802088. URL http://dx.doi.org/10.1063/1.4802088

[89] Chen Y, Zopf M, Keil R, Ding F, Schmidt OG, Highly-efficient extraction of entangled photons from quantum dots using a broadband optical antenna. Nature Communications 9(1) (2018). DOI 10.1038/ s41467-018-05456-2. URL http://dx.doi.org/10.1038/s41467-018-05456-2

[90] Liu J, Su R, Wei Y, Yao B, Silva SFCd, Yu Y, Iles-Smith J, Srinivasan K, Rastelli A, Li J, et al., A solid-state source of strongly entangled photon pairs with high brightness and indistinguishability. Nature Nanotechnology (2019). DOI 10.1038/s41565-019-0435-9. URL http://dx.doi.org/10.1038/ s41565-019-0435-9

[91] Yamamoto Y, Slusher RE, Optical processes in microcavities. Physics Today 46(6), 66 (1993). DOI 10.1063/ 1.881356. URL http://dx.doi.org/10.1063/1.881356

[92] Guo X, Zhou X, Wang JH, Luo ZJ, Zhou Q, Liu K, Hu MZ, Ding Z, Critical surface phase of 2(2E4) reconstructed zig-zag chains on inas(001). Thin Solid Films 562, 326 (2014). DOI https://doi. org/10.1016/j.tsf.2014.02.116. URL http://www.sciencedirect.com/science/article/pii/ S0040609014002934

[93] Gschrey M, Gericke F, Schüßler A, Schmidt R, Schulze JH, Heindel T, Rodt S, Strittmatter A, Reitzenstein S, In situ electron-beam lithography of deterministic single-quantum-dot mesa-structures using low-temperature cathodoluminescence spectroscopy. Applied Physics Letters 102(25), 251113 (2013). DOI 10.1063/1.4812343. URL http://dx.doi.org/10.1063/1.4812343

[94] Dousse A, Lanco L, Suffczyński J, Semenova E, Miard A, Lemaître A, Sagnes I, Roblin C, Bloch J, Senellart $\mathrm{P}$, Controlled light-matter coupling for a single quantum dot embedded in a pillar microcavity using farfield optical lithography. Phys. Rev. Lett. 101, 267404 (2008). DOI 10.1103/PhysRevLett.101.267404. URL https://link.aps.org/doi/10.1103/PhysRevLett.101.267404

[95] Kistner C, Heindel T, Schneider C, Rahimi-Iman A, Reitzenstein S, Höfling S, Forchel A, Demonstration of strong coupling via electro-optical tuning in high-quality qd-micropillar systems. Opt. Express 16(19), 15006 (2008). DOI 10.1364/oe.16.015006. URL http://dx.doi.org/10.1364/OE.16.015006

[96] Kojima T, Kojima K, Asano T, Noda S, Accurate alignment of a photonic crystal nanocavity with an embedded quantum dot based on optical microscopic photoluminescence imaging. Applied Physics Letters 102(1), 011110 (2013). DOI 10.1063/1.4773882. URL http://dx.doi.org/10.1063/1.4773882

[97] Badolato A, Hennessy K, Atatüre M, Dreiser J, Hu E, Petroff P M, Imamoğlu A, Deterministic coupling of single quantum dots to single nanocavity modes. Science 308(5725), 1158 (2005)

[98] Thon SM, Rakher MT, Kim H, Gudat J, Irvine WTM, Petroff PM, Bouwmeester D, Strong coupling through optical positioning of a quantum dot in a photonic crystal cavity. Appl. Phys. Lett. 94(11), 111115 (2009). DOI 10.1063/1.3103885. URL http://dx.doi.org/10.1063/1.3103885 
[99] Notomi M, Manipulating light with strongly modulated photonic crystals. Reports on Progress in Physics 73(9), 096501 (2010). DOI 10.1088/0034-4885/73/9/096501. URL http://dx.doi.org/10.1088/ $0034-4885 / 73 / 9 / 096501$

[100] Hijlkema M, Weber B, Specht HP, Webster SC, Kuhn A, Rempe G, A single-photon server with just one atom. Nat. Phys. 3(4), 253 (2007). DOI 10.1038/nphys569

[101] Kurtsiefer C, Mayer S, Zarda P, Weinfurter H, Stable solid-state source of single photons. Phys. Rev. Lett. 85(2), 290 (2000). URL http://www.ncbi.nlm.nih.gov/pubmed/10991265

[102] Holmes MJ, Choi K, Kako S, Arita M, Arakawa Y, Room-temperature triggered single photon emission from a III-nitride site-controlled nanowire quantum dot. Nano Lett. 14(2), 982 (2014). DOI 10.1021/nl404400d

[103] Jarjour AF, Taylor RA, Oliver RA, Kappers MJ, Humphreys CJ, Tahraoui A, Cavity-enhanced blue singlephoton emission from a single InGaNGaN quantum dot. Appl. Phys. Lett. 91(5), 052101 (2007). DOI 10.1063/ 1.2767217. URL http://link.aip.org/link/APPLAB/v91/i5/p052101/s1\{\&\}Agg=doi

[104] Deshpande S, Frost T, Hazari A, Bhattacharya P, Electrically pumped single-photon emission at room temperature from a single InGaN/GaN quantum dot. Appl. Phys. Lett. 105(14) (2014). DOI 10.1063/1.4897640

[105] Jarjour A, Oliver R, Tahraoui A, Kappers M, Humphreys C, Taylor R, Control of the Oscillator Strength of the Exciton in a Single InGaN-GaN Quantum Dot. Phys. Rev. Lett. 99(19), 1 (2007). DOI 10.1103/PhysRevLett. 99.197403. URL http://link.aps.org/doi/10.1103/PhysRevLett.99.197403

[106] Reid BP, Kocher C, Zhu T, Oehler F, Chan CC, Oliver RA, Taylor RA, Non-polar InGaN quantum dot emission with crystal-axis oriented linear polarization. Appl. Phys. Lett. 106(17) (2015). DOI 10.1063/1.4919656

[107] Wang T, Puchtler TJ, Patra SK, Zhu T, Ali M, Badcock TJ, Ding T, Oliver RA, Schulz S, Taylor RA, Direct generation of linearly polarized single photons with a deterministic axis in quantum dots. Nanophotonics $6(5)$, 1175 (2017). DOI 10.1515/nanoph-2017-0027

[108] Waks E, Inoue K, Santori C, Fattal D, Vuckovic J, Solomon G, Zhang B, Yamamoto Y, Quantum cryptography with a photon turnstile device. Extended Abstracts of the 2002 International Conference on Solid State Devices and Materials (2002). DOI 10.7567/ssdm.2002.f-9-1. URL http://dx.doi.org/10.7567/SSDM. $2002 \cdot F-9-1$

[109] Bretagnon T, Lefebvre P, Valvin P, Bardoux R, Guillet T, Taliercio T, Gil B, Grandjean N, Semond F, Damilano B, Dussaigne A, Massies J, Radiative lifetime of a single electron-hole pair in GaNAlN quantum dots. Phys. Rev. B 73(11), 113304 (2006). DOI 10.1103/PhysRevB.73.113304. URL https://Iink.aps.org/ doi/10.1103/PhysRevB.73.113304

[110] Reid BPL, Zhu T, Chan CCS, Kocher C, Oehler F, Emery R, Kappers MJ, Oliver RA, Taylor RA, High temperature stability in non-polar (110) InGaN quantum dots: Exciton and biexciton dynamics. physica status solidi (c) 11(3-4), 702 (2014). DOI 10.1002/pssc.201300666. URL http:// doi .wiley •com/10.1002/pssc. 201300666

[111] Kako S, Santori C, Hoshino K, Götzinger S, Yamamoto Y, Arakawa Y, A gallium nitride single-photon source operating at 200K. Nat. Mater. 5(11), 887 (2006). DOI 10.1038/nmat1763

[112] Arita M, Le Roux F, Holmes MJ, Kako S, Arakawa Y, Ultraclean single photon emission from a gan quantum dot. Nano Lett. 17(5), 2902 (2017). DOI 10.1021/acs.nanolett.7b00109. URL http: / / dx. doi .org/10 . 1021 /acs.nanolett. 7b00109

[113] Holmes MJ, Kako S, Choi K, Arita M, Arakawa Y, Single Photons from a Hot Solid-State Emitter at $350 \mathrm{~K}$. ACS Photonics 3(4), 543 (2016). DOI 10.1021/acsphotonics.6b00112 
[114] Zhou Y, Wang Z, Rasmita A, Kim S, Berhane A, Bodrog Z, Adamo G, Gali A, Aharonovich I, Gao Wb, Room temperature solid-state quantum emitters in the telecom range. Sci. Adv. 4(3) (2018)

[115] Kanta Patra S, Wang T, Puchtler TJ, Zhu T, Oliver RA, Taylor RA, Schulz S, Theoretical and experimental analysis of radiative recombination lifetimes in nonpolar InGaN/GaN quantum dots. Physica Status Solidi (B) Basic Research 254(8) (2017). DOI 10.1002/pssb.201600675

[116] Wang T, Puchtler TJ, Zhu T, Jarman JC, Nuttall LP, Oliver RA, Taylor RA, Polarisation-controlled single photon emission at high temperatures from InGaN quantum dots. Nanoscale 9(27), 9421 (2017). DOI 10.1039/ C7NR03391E. URL http://xlink.rsc.org/?DOI=C7NR03391E

[117] Ga Ž, Holmes M, Chernysheva E, Müller M, Veit P, Bertram F, Christen J, María J, Calbet G, Arakawa Y, Calleja E, Lazi S, Emission of linearly polarized single photons from quantum dots contained in nonpolar, semipolar and polar sections of pencil-like InGaN / GaN nanowires. ACS Photonics (2017). DOI 10.1021/ acsphotonics.6b01030

[118] Kindel C, Kako S, Kawano T, Oishi H, Arakawa Y, Collinear Polarization of Exciton / Biexciton Photoluminescence from Single Hexagonal GaN Quantum Dots. Japanese Journal of Applied Physics 48, 04C116 (2009). DOI 10.1143/JJAP.48.04C116

[119] Sergent S, Kako S, Burger M, Schupp T, As DJ, Arakawa Y, Polarization properties of single zinc-blende gan/aln quantum dots. Phys. Rev. B 90, 235312 (2014). DOI 10.1103/PhysRevB.90.235312. URL https: //link.aps.org/doi/10.1103/PhysRevB.90.235312

[120] Lundskog A, Hsu CW, Fredrik Karlsson K, Amloy S, Nilsson D, Forsberg U, Olof Holtz P, Janzén E, Direct generation of linearly polarized photon emission with designated orientations from site-controlled ingan quantum dots. Light: Science \& Applications 3(1), e139 (2014). DOI 10.1038/lsa.2014.20. URL http://dx.doi.org/10.1038/1sa.2014.20

[121] Teng Ch, Zhang L, Hill TA, Demory B, Deng H, Ku Pc, Elliptical quantum dots as on-demand single photons sources with deterministic polarization states. Appl. Phys. Lett. 191105(2015) (2016). DOI 10.1063/1.4935463

[122] Kocher CC, Puchtler TJ, Jarman JC, Zhu T, Wang T, Nuttall L, Oliver RA, Taylor RA, Highly polarized electrically driven single-photon emission from a non-polar InGaN quantum dot. Appl. Phys. Lett. 111(25) (2017). DOI $10.1063 / 1.5008720$

[123] Mendelson N, Xu ZQ, Tran TT, Kianinia M, Scott J, Bradac C, Aharonovich I, Toth M, Engineering and tuning of quantum emitters in few-layer hexagonal boron nitride. ACS Nano 13(3), 3132 (2019). DOI 10.1021/ acsnano.8b08511. URL http://dx.doi .org/10.1021/acsnano.8b08511

[124] Bourrellier R, Meuret S, Tararan A, Stéphan O, Kociak M, Tizei LHG, Zobelli A, Bright uv single photon emission at point defects in h-bn. Nano Lett. 16(7), 4317 (2016). DOI 10.1021/acs.nanolett.6b01368. URL http://dx.doi.org/10.1021/acs.nanolett.6b01368

[125] Martínez LJ, Pelini T, Waselowski V, Maze JR, Gil B, Cassabois G, Jacques V, Efficient single photon emission from a high-purity hexagonal boron nitride crystal. Phys. Rev. B 94, 121405 (2016). DOI 10.1103/PhysRevB. 94.121405. URL https://link.aps.org/doi/10.1103/PhysRevB.94.121405

[126] Vogl T, Campbell G, Buchler BC, Lu Y, Lam PK, Fabrication and deterministic transfer of high-quality quantum emitters in hexagonal boron nitride. ACS Photonics 5(6), 2305 (2018). DOI 10.1021/acsphotonics.8b00127. URL http://dx.doi.org/10.1021/acsphotonics.8b00127

[127] Kumar A, Low T, Fung KH, Avouris P, Fang NX, Tunable light-matter interaction and the role of hyperbolicity in graphene-hbn system. Nano Lett. 15(5), 3172 (2015). DOI 10.1021/acs.nanolett.5b01191. URL http: //dx.doi.org/10.1021/acs.nanolett.5b01191 
[128] Tawfik SA, Ali S, Fronzi M, Kianinia M, Tran TT, Stampfl C, Aharonovich I, Toth M, Ford MJ, Firstprinciples investigation of quantum emission from hbn defects. Nanoscale 9(36), 13575 (2017). DOI 10.1039/c7nr04270a. URL http://dx.doi.org/10.1039/C7NR04270A

[129] Vil'k YN, Chupov VD, ShvaikoShvaikovskii VE, Garshin AP, A theoretical analysis of the formation of nonstoichiometric defects in hexagonal boron nitride. Refract. Ind. Ceram 42(3/4), 146 (2001). DOI 10.1023/a: 1011384129992. URL http://dx.doi.org/10.1023/A:1011384129992

[130] Grosso G, Moon H, Lienhard B, Ali S, Efetov DK, Furchi MM, Jarillo-Herrero P, Ford MJ, Aharonovich I, Englund D, Tunable and high-purity room temperature single-photon emission from atomic defects in hexagonal boron nitride. Nat. Commun. 8(1) (2017). DOI 10.1038/s41467-017-00810-2. URL http: //dx.doi.org/10.1038/s41467-017-00810-2

[131] Tran TT, Elbadawi C, Totonjian D, Lobo CJ, Grosso G, Moon H, Englund DR, Ford MJ, Aharonovich I, Toth M, Robust multicolor single photon emission from point defects in hexagonal boron nitride. ACS Nano 10(8), 7331 (2016). DOI 10.1021/acsnano.6b03602. URL http://dx.doi.org/10.1021/acsnano.6b03602

[132] Jana M, Singh RN, Progress in cvd synthesis of layered hexagonal boron nitride with tunable properties and their applications. Int. Mater. Rev. 63(3), 162 (2017). DOI 10.1080/09506608.2017.1322833. URL http: //dx.doi.org/10.1080/09506608.2017.1322833

[133] Loredo JC, Zakaria NA, Somaschi N, Anton C, de Santis L, Giesz V, Grange T, Broome MA, Gazzano O, Coppola G, et al., Scalable performance in solid-state single-photon sources. Optica 3(4), 433 (2016). DOI 10.1364/optica.3.000433. URL http://dx.doi.org/10.1364/OPTICA.3.000433

[134] Mnaymneh K, Dalacu D, McKee J, Lapointe J, Haffouz S, Poole PJ, Aers GC, Williams RL, Monolithic integration of single photon sources via evanescent coupling of tapered inp nanowires to sin waveguides. arXiv:1901.00469v1 [physics.app-ph] (2018)

[135] Sangouard N, Simon C, de Riedmatten H, Gisin N, Quantum repeaters based on atomic ensembles and linear optics. Rev. Mod. Phys. 83, 33 (2011). DOI 10.1103/RevModPhys.83.33. URL https : / I ink . aps . org / doi/10.1103/RevModPhys.83.33

[136] Weber JH, Kambs B, Kettler J, Kern S, Maisch J, Vural H, Jetter M, Portalupi SL, Becher C, Michler P, Twophoton interference in the telecom c-band after frequency conversion of photons from remote quantum emitters. Nature Nanotechnology 14(1), 23 (2019). DOI 10.1038/s41565-018-0279-8. URL http: / / dx. doi .org/ $10.1038 / \mathrm{s} 41565-018-0279-8$

[137] Loredo JC, Broome MA, Hilaire P, Gazzano O, Sagnes I, Lemaitre A, Almeida MP, Senellart P, White AG, Boson sampling with single-photon fock states from a bright solid-state source. Phys. Rev. Lett. 118, 130503 (2017). DOI 10.1103/PhysRevLett.118.130503. URL https://link.aps.org/doi/10. 1103 /PhysRevLett.118.130503

[138] He Y, Ding X, Su ZE, Huang HL, Qin J, Wang C, Unsleber S, Chen C, Wang H, He YM, Wang XL, Zhang WJ, Chen SJ, Schneider C, Kamp M, You LX, Wang Z, Höfling S, Lu CY, Pan JW, Time-bin-encoded boson sampling with a single-photon device. Phys. Rev. Lett. 118, 190501 (2017). DOI 10.1103/PhysRevLett.118. 190501. URL https://link.aps.org/doi/10.1103/PhysRevLett.118.190501

[139] Chang DE, Cirac JI, Kimble HJ, Self-organization of atoms along a nanophotonic waveguide. Phys. Rev. Lett. 110, 113606 (2013). DOI 10.1103/PhysRevLett.110.113606. URL https://link.aps.org/doi/10 . 1103 /PhysRevLett.110.113606 
[140] Cho JH, Kim YM, Lim SH, Yeo HS, Kim S, Gong SH, Cho YH, Strongly coherent single-photon emission from site-controlled ingan quantum dots embedded in gan nanopyramids. ACS Photonics 5(2), 439 (2018). DOI 10.1021/acsphotonics.7b00922. URL http://dx.doi.org/10.1021/acsphotonics.7b00922

[141] Carmesin C, Olbrich F, Mehrtens T, Florian M, Michael S, Schreier S, Nawrath C, Paul M, Höschele J, Gerken B, Kettler J, Portalupi SL, Jetter M, Michler P, Rosenauer A, Jahnke F, Structural and optical properties of inas/(in)gaas/gaas quantum dots with single-photon emission in the telecom c-band up to $77 \mathrm{k}$. Phys. Rev. B 98, 125407 (2018). DOI 10.1103/PhysRevB.98.125407. URL https://link.aps.org/doi/10. 1103 /PhysRevB.98.125407 